ARTICLE

https://doi.org/10.1038/s41467-019-10590-6

\title{
Native top-down mass spectrometry provides insights into the copper centers of membrane- bound methane monooxygenase
}

Soo Y. Ro1,2, Luis F. Schachner (1) 1,2, Christopher W. Koo', Rahul Purohit', Jonathan P. Remis ${ }^{1}$, Grace E. Kenney (1) ${ }^{1}$, Brandon W. Liauw ${ }^{1}$, Paul M. Thomas (1) ${ }^{1}$, Steven M. Patrie ${ }^{1}$, Neil L. Kelleher (1) ${ }^{1} \&$ Amy C. Rosenzweig (1) ${ }^{1}$

Aerobic methane oxidation is catalyzed by particulate methane monooxygenase (pMMO), a copper-dependent, membrane metalloenzyme composed of subunits PmoA, PmoB, and PmoC. Characterization of the copper active site has been limited by challenges in spectroscopic analysis stemming from the presence of multiple copper binding sites, effects of detergent solubilization on activity and crystal structures, and the lack of a heterologous expression system. Here we utilize nanodiscs coupled with native top-down mass spectrometry (nTDMS) to determine the copper stoichiometry in each pMMO subunit and to detect post-translational modifications (PTMs). These results indicate the presence of a mononuclear copper center in both $\mathrm{PmoB}$ and $\mathrm{PmoC}$. pMMO-nanodisc complexes with a higher stoichiometry of copper-bound PmoC exhibit increased activity, suggesting that the PmoC copper site plays a role in methane oxidation activity. These results provide key insights into the pMMO copper centers and demonstrate the ability of nTDMS to characterize complex membrane-bound metalloenzymes.

\footnotetext{
${ }^{1}$ Departments of Molecular Biosciences and of Chemistry, Northwestern University, 2205 Tech Drive, Evanston, IL 60208, USA. ${ }^{2}$ These authors contributed equally: Soo Y. Ro, Luis F. Schachner. Correspondence and requests for materials should be addressed to N.L.K. (email: n-kelleher@northwestern.edu) or to A.C.R. (email: amyr@northwestern.edu)
} 
articulate methane monooxygenase (pMMO) is an integral membrane metalloenzyme that oxidizes methane to methanol $^{1}$ in methanotrophic bacteria ${ }^{2}$. pMMO comprises three subunits, $\mathrm{PmoB}, \mathrm{PmoA}$, and $\mathrm{PmoC}$, assembled into a larger $\alpha_{3} \beta_{3} \gamma_{3}$ complex ${ }^{3-7}$. The enzymatic activity of pMMO depends on the presence of copper, with approximately two copper ions per $\alpha \beta \gamma$ protomer required for optimal activity ${ }^{3,8}$. Extensive efforts have been devoted to elucidating the nature of the pMMO copper active site ${ }^{1,9}$, with the ultimate goal of identifying the reactive copper-oxygen intermediate responsible for activating the 105 $\mathrm{kcal} / \mathrm{mol} \mathrm{C}-\mathrm{H}$ bond in methane ${ }^{1}$. A molecular and mechanistic understanding of the pMMO active site is essential for the design of methane remediation tools, including synthetic catalysts and engineered methanotrophs ${ }^{10,11}$, and may also provide insight into copper-mediated oxidation chemistry.

Candidate locations for the copper active site were first identified in the crystal structure of Methylococcus capsulatus (Bath) pMMO (Bath-pMMO), which revealed three metal centers ${ }^{6}$. Two copper centers were modeled in PmoB: a nonconserved monocopper site ligated by His 48 and His 72 (bis-His site) that is not observed in other pMMO structures, and a conserved site at the amino terminus ligated by $\mathrm{His} 33$, His 137 , and $\mathrm{His} 139\left(\mathrm{Cu}_{\mathrm{B}}\right.$ site). The latter site was initially modeled as dicopper on the basis of extended X-ray absorption fine structure (EXAFS) data ${ }^{3,5,12}$, but later analysis and crystal structures indicated that this site may instead be monocopper (Fig. 1) ${ }^{3,4,13}$. In addition, a third site occupied by zinc from the crystallization buffer was present in the PmoC subunit with ligands Asp 127, His 131, and His 144. This site, sometimes called the variable metal binding site, can also be occupied by copper ${ }^{3}$ and is located in a chronically disordered region of the PmoC subunit ${ }^{4}$.

The nuclearity of the $\mathrm{Cu}_{\mathrm{B}}$ site has been defined unambiguously by recent in vivo advanced electron paramagnetic resonance

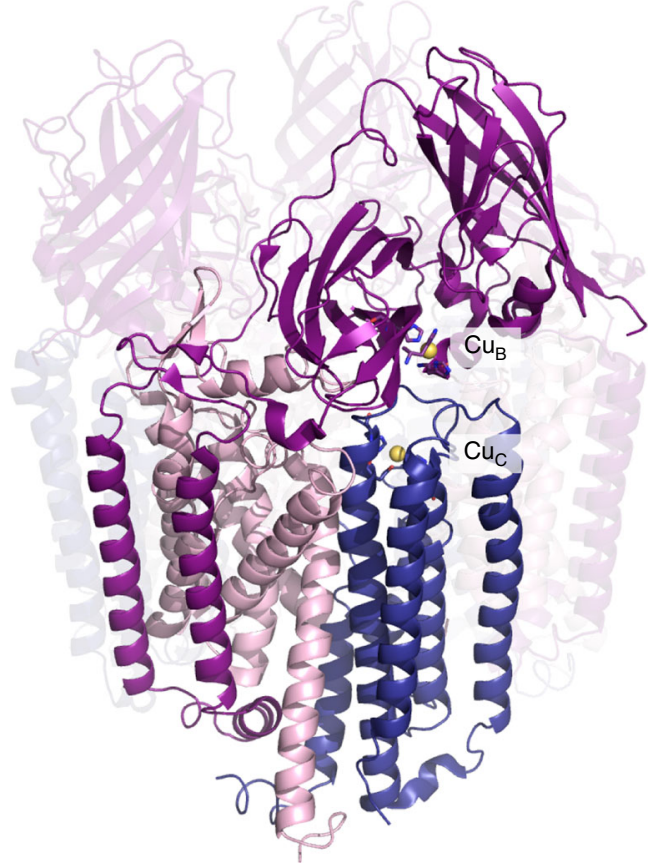

Fig. 1 The pMMO structure and location of the metal centers. In the crystal structure of Methylocystis sp. strain Rockwell-pMMO (4PHZ), there is a monocopper center in PmoB coordinated by residues His 29, His 133, and His $135\left(\mathrm{Cu}_{\mathrm{B}}\right)$, and a monocopper center in PmoC coordinated by Asp 129, His 133, and His $146\left(\mathrm{Cu}_{\mathrm{C}}\right)$. PmoA, PmoB, and PmoC are shown in pink, purple, and blue, respectively, with one of the three protomers highlighted. Copper ions are shown as yellow spheres
(EPR) spectroscopic characterization of Methylococcus capsulatus (Bath). In addition, the presence of a second monocopper center at the PmoC variable metal binding site, denoted the $\mathrm{Cu}_{\mathrm{C}}$ site, was demonstrated using double electron-electron resonance (DEER) spectroscopy ${ }^{14}$. These data established an important correlation between the sites observed in the crystal structure and the sites present in the cell. While the $\mathrm{Cu}_{\mathrm{B}}$ site was previously assigned as the active site ${ }^{8}$, our more recent studies indicate that it is not sufficient for methane oxidation ${ }^{14}$, consistent with the requirement for two copper ions ${ }^{3,8}$. The possibility that methane oxidation occurs at the $\mathrm{PmoC} \mathrm{Cu}_{\mathrm{C}}$ site has been raised ${ }^{14}$, but lacks direct experimental support.

The study of pMMO has been hindered by the limitations of traditional biochemical, structural, and spectroscopic approaches. Metal analyses indicating the presence of 2-3 copper ions provide no insight into the specific locations of these metal ions, necessitating inferences based on combined crystallography and spectroscopy. The crystal structures are subject to artifacts from the crystallization buffer, such as the presence of zinc in the PmoC site ${ }^{5,6}$ as well as unknown effects of detergent solubilization and the crystallization process. In addition, some flexible regions are never observed in the electron density maps ${ }^{4}$. Spectroscopic data collected on pMMO reflect a mixture of copper species, rendering it nontrivial and in the case of EXAFS, impossible, to separate signals arising from different sites. This issue is compounded by the fact that pMMO has not been expressed heterologously, precluding facile sitedirected mutagenesis. These challenges are not specific to pMMO; determination of metal stoichiometry and localization can be a major challenge for large, multisubunit metalloprotein complexes.

An emerging alternative approach for metal center characterization is native mass spectrometry (nMS), which typically employs electrospray ionization (ESI) at neutral $\mathrm{pH}$ from volatile, non-reducing buffers ${ }^{15}$, and instrument settings that faithfully preserve the primary and quaternary composition of complexes in the sample ${ }^{16,17}$. Coupling tandem MS (MS ${ }^{n}$ activation of a non-covalent protein assembly to the nMS analysis ${ }^{18}$ can help to characterize liberated components from the complex, such as subunits ${ }^{18}$. Moreover, measurement of intact mass values by nMS followed by gas-phase protein fragmentation, termed native topdown mass spectrometry (nTDMS) ${ }^{19,20}$, enables the identification and characterization of specific proteoforms emanating from encoding genes including those containing underlying sequence changes due to polymorphisms, unexpected truncations, or posttranslational modification (PTM) ${ }^{19-24}$. In particular, nTDMS can be used to determine metal stoichiometry of each subunit and even the identities of the metal binding ligands ${ }^{25-27}$. In $2013^{19}$, a nTDMS platform achieved a three-tiered tandem mass spectrometry (MS) process, comprising measurement of an intact protein complex $\left(\mathrm{MS}^{1}\right)$, analysis of ejected monomer(s) $\left(\mathrm{MS}^{2}\right)$, and backbone fragmentation of each monomer $\left(\mathrm{MS}^{3}\right.$, or pseudo-MS $\left.{ }^{3}\right)$ measured at isotopic resolution (Fig. 2) ${ }^{19,20}$.

Here we apply nTDMS to pMMO. While some membrane proteins have been characterized by $\mathrm{nMS}^{28-31}$, this study involves nTDMS characterization of a multisubunit, membrane metalloenzyme via $\mathrm{MS}^{3}$ fragmentation of individual subunits for metal ion identification, localization, and stoichiometric determination. Our nTDMS analysis of pMMO in micelles and nanodiscs ${ }^{32}$ identifies proteoforms of pMMO subunits and PTMs that may have functional implications. Most importantly, the data support the presence of one copper ion each in the $\mathrm{PmoB}$ and PmoC subunits, and in combination with activity profiles of pMMO in nanodiscs, suggest that the copper ion bound to PmoC is important for the oxidation of methane to methanol. 

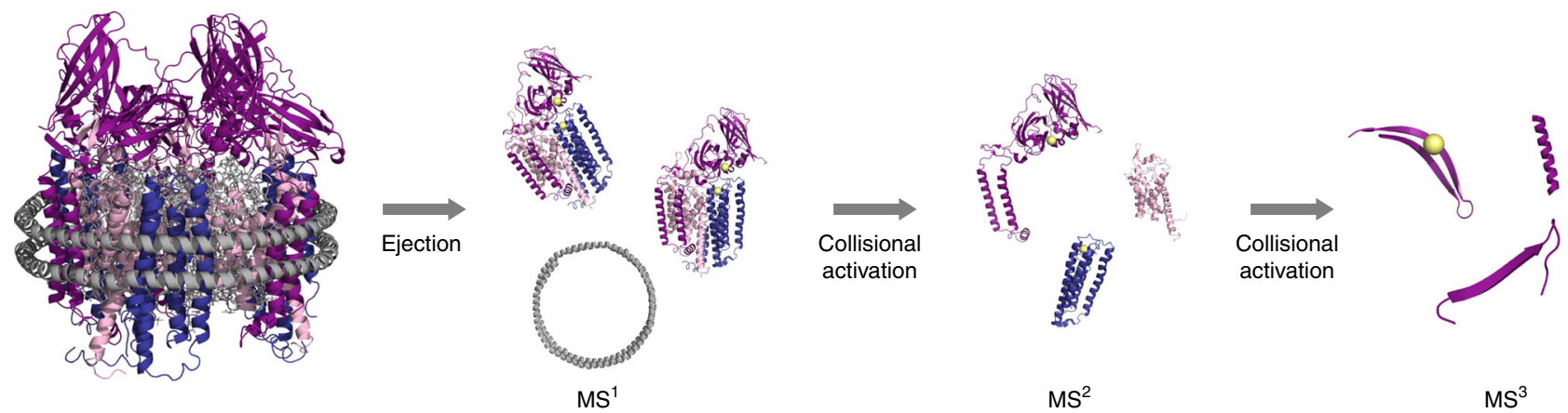

Fig. 2 The nTDMS platform for pMMO characterization. The pMMO-nanodisc complex is subjected to ejection from the nanodisc using collision-induced dissociation (CID) at the source resulting in the stabilization of the pMMO protomer species $\left(M^{1}\right)$. Increasing collisional activation breaks up the protomer into individual pMMO subunits $\left(\mathrm{MS}^{2}\right)$. Further collisional activation enables backbone fragmentation of each subunit using higher energy collisional dissociation (HCD) in the HCD cell of the instrument $\left(\mathrm{MS}^{3}\right.$ )

\section{Results}

Defining the proteoform composition of pMMO by nTDMS. Our initial nTDMS studies focused on pMMO from Methylomicrobium alcaliphilum 20Z (20Z-pMMO). Methanotrophs from the genus Methylomicrobium ( $\mathrm{Mm}$.) have attracted interest as tractable model systems in engineering applications for methaneto-biofuel conversion, and $\mathrm{Mm}$. alcaliphilum $20 \mathrm{Z}$ has been the subject of several studies ${ }^{33-35}$, including the recent characterization of its $\mathrm{pMMO}^{4}$. In the crystal structure of 20Z-pMMO, one copper ion was modeled into the $\mathrm{Cu}_{\mathrm{B}}$ site, supported by EXAFS analysis. However, metal quantitation and EPR analysis indicated the presence of two copper ions per pMMO protomer ${ }^{4}$. In the crystal structure, the $\mathrm{PmoB}$ bis-His site is unoccupied and the PmoC subunit is mostly disordered, rendering it impossible to determine if it houses any metal ions. Thus, additional data are needed to assess the metal centers in 20Z-pMMO, motivating its investigation by nTDMS.

20Z-pMMO was solubilized from as-isolated membranes and purified by anion exchange chromatography ${ }^{4}$. After reconstituting 20Z-pMMO in Triton X-100 micelles, the complex was subjected to nMS analysis for characterization. The MS ${ }^{1}$ analysis (Supplementary Fig. 1a) produced a species exhibiting a 15-18+ charge state distribution. Deconvolution of the charge states of the major species present at 93\% (Supplementary Fig. 1a, labeled in purple) yielded a mass of $98,696.0 \pm 1.1 \mathrm{Da}$ ( \pm represents standard deviation from the reported average mass calculated by sampling multiple charge states of the protein species) (Fig. 3a). The theoretical mass of an unmodified pMMO protomer, 99,255.7 $\mathrm{Da}$, is based on the amino acid sequences of the subunits, assuming the cleavage of a known signal peptide at the PmoB N-terminus ${ }^{36}$ (theoretical mass of a protomer with an uncleaved PmoB is $102,638.7 \mathrm{Da})$. While the observed mass of the predominant species is lower than the theoretical mass that assumes cleavage of the $\mathrm{PmoB}$ signal peptide (mass difference, $\Delta \mathrm{m}=-559.7 \mathrm{Da}$ ), the close match suggests that the species ejected from the Triton X-100 micelle is a modified pMMO protomer.

To investigate the possible presence of PTMs and metal cofactors in proteoforms comprising the pMMO protomer, the $16+$ charge state of the pMMO-micelle complex was subjected to nTDMS. First, intact pMMO protomer was activated by collisions with neutral gas in the ESI source region to produce a pseudo$\mathrm{MS}^{2}$ of the ejected subunits (Supplementary Fig. 1b). Three major protein species that correspond closely in mass to each one of the pMMO subunits were detected (Fig. 3b). The PmoA subunit, with a theoretical mass of $28,257.1 \mathrm{Da}$, was tentatively assigned to the $28,166.8 \pm 0.4 \mathrm{Da}$ proteoform, with a $\Delta \mathrm{m}$ of $-89.1 \mathrm{Da}$ consistent within a dalton for the removal of the initiator methionine
$\left(\mathrm{Met}_{\mathrm{OFF}}\right)$ and the addition of N-terminal acetylation (NtAc) to the new N-terminus. The $42,221.6 \pm 0.3 \mathrm{Da}$ proteoform, which is closest in mass to PmoB (theoretical mass $42,147.17 \mathrm{Da}, \Delta \mathrm{m}=$ $+74.4 \mathrm{Da})$, could result from the replacement of two protons by one copper(II) ion ${ }^{4}(+61.5 \mathrm{Da})$ and a potential PTM. The third species had a mass of $28,245.2 \pm 0.5 \mathrm{Da}$, which may be attributable to a truncated proteoform of PmoC (theoretical mass $28,851.4 \mathrm{Da}, \Delta \mathrm{m}=-606.2 \mathrm{Da}$ ). The addition of the three detected masses yields an $\alpha \beta \gamma$ protomer of 98,633.6 Da, which is 62.4 Da smaller than the observed protomer via $\mathrm{MS}^{1}$. This mass loss suggests that a copper ion (theoretical average mass of 61.5 $\mathrm{Da})$ may be lost upon subunit ejection from the pMMO-micelle complex.

To confirm subunit assignments and characterize their mass shifts, intact proteoforms need to be fragmented by tandem MS. Attempts at tandem MS on subunits ejected from the Triton X100 micelle were unsuccessful due to the limited ability to activate ions in the ESI source; for example, disrupting the micelle to produce intact $\mathrm{pMMO}$ ions required relatively high settings of $150-195 \mathrm{~V}$. This precluded further activation of the complex in the ESI source $(200 \mathrm{~V} \max )$, which is essential for individual subunits to be isolated and further dissociated in the higher energy collision dissociation cell (HCD) within the instrument.

nTDMS analysis of pMMO proteoforms ejected from nanodiscs. To achieve further collisional activation and to potentially stabilize the copper ion lost upon subunit ejection, Triton X-100 micelles were substituted with nanodiscs, discoidal lipid bilayers absent of detergent and commonly used to stabilize membrane proteins ${ }^{37}$. Nanodiscs have been reported to minimize coulombinduced unfolding in the gas phase, suggesting that they can be protective of labile non-covalent interactions upon ejection from the assembly ${ }^{29-31}$. 20Z-pMMO was embedded in nanodiscs using POPC lipids and the membrane scaffold proteins MSP2N2 or MSP1E3D1 (Supplementary Figs 2-3) ${ }^{32}$. Nanodiscs formed using MSP2N2 and MSP1E3D1 have diameter distances of up to 17 and $12 \mathrm{~nm}$, respectively, and can accommodate the $9 \mathrm{~nm}$ pMMO complex. MSP1E3D1 provided higher reconstitution yields and stability than MSP2N2, potentially because the smaller diameter of MSP1E3D1 allows for a tighter fit with pMMO. Twodimensional class averages of cryo-electron microscopy images (cryo-EM) and SDS-PAGE were used to assess reconstitution and confirm the presence of all three pMMO subunits in MSP2N2 nanodiscs (Fig. 4, Supplementary Fig. 4); MSP1E3D1 nanodiscs were analyzed by negative stain electron microscopy images and SDS-PAGE.

The 20Z-pMMO nanodisc complex was subjected to the nTDMS platform, which presented some initial challenges. First, 


\section{Deconvoluted MS ${ }^{1}$ and MS²: 20Z-pMMO protomer ejected from micelle}

a
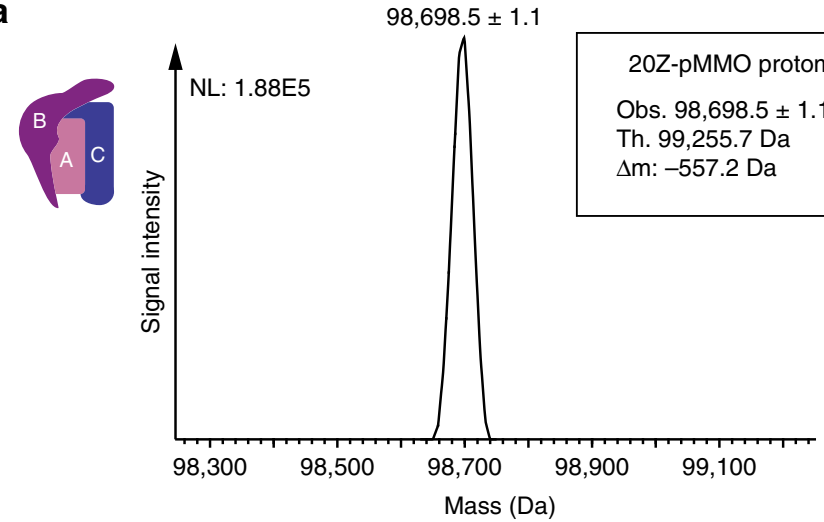

PmoA
Obs. $28,166.8 \pm 0.4 \mathrm{Da}$
Th. $28,257.1 \mathrm{Da}$
$\Delta \mathrm{m}:-90.3 \mathrm{Da}$

PmoB
Obs. $42,221.6 \pm 0.3 \mathrm{Da}$
Th. $42,147.17 \mathrm{Da}$
$\Delta \mathrm{m}: 74.4 \mathrm{Da}$
PmoC
Obs. $28,245.2 \pm 0.5 \mathrm{Da}$
Th. $28,851.44$
$\Delta \mathrm{m}:-606.24 \mathrm{Da}$

而

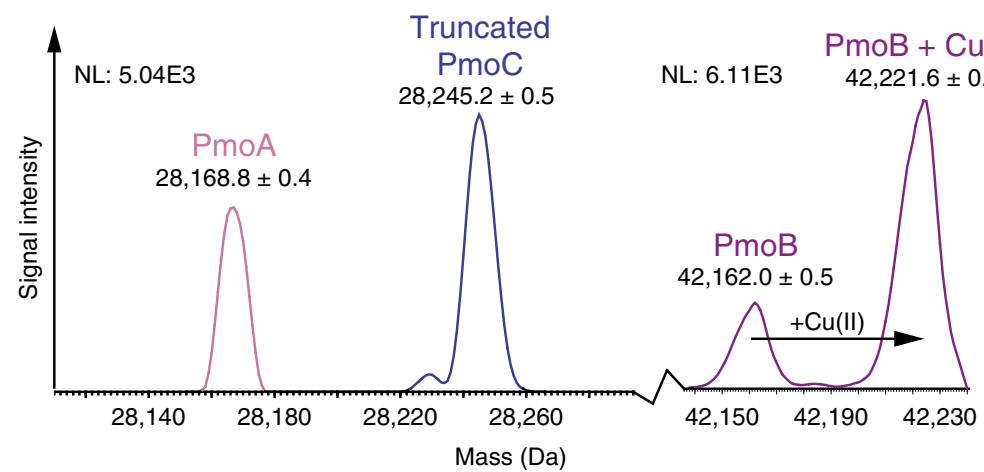

Fig. 3 nTDMS analysis of 20Z-pMMO in Triton X-100 micelles. a Deconvoluted MS1 of 20Z-pMMO protomer upon ejection from a Triton X-100 micelle. Charge state deconvolution of the major species yields a mass of $98,696.5 \pm 1.1 \mathrm{Da}$. The theoretical mass is derived from the unmodified subunits of pMMO and accounts for the cleavage of a known signal peptide in PmoB. b Deconvoluted MS² of 20Z-pMMO subunits ejected from the 16+ charge state of the protomer after activation by collisions with neutral gas. The spectrum shows detection of three species, labeled pink, purple, and blue and assigned to PmoA, PmoB, and PmoC, respectively. Adding the measured masses of the ejected subunits yields 98,633.6 Da, which is $62.4 \mathrm{Da}$ smaller than the major protomer mass measured in the $\mathrm{MS}^{1}$. NL values reflect maximum signal intensity in the spectrum

we were unable to obtain an intact mass of the complex given the heterogeneity of the signals detected. Second, excess of lipids in the nanodiscs led to the detection of lipid clusters, which dominated the signal in $\mathrm{MS}^{1}$ spectra. We overcame these difficulties by titrating the lipid content in the nanodiscs to an optimal concentration that reduced signals from lipid clusters, as measured by nMS, while still preserving enzymatic activity. Additionally, we tuned the ion optics of the mass spectrometer, including the C-trap entrance lens voltage, to filter out high intensity lipid cluster ions. The pMMO complex was subjected to subunit ejection from the nanodisc complex using collisioninduced dissociation (CID) at the source. The successful ejection of the individual subunits (Supplementary Fig. 5) with $195 \mathrm{eV}$ of source fragmentation enabled their subsequent isolation by the quadrupole mass filter and fragmentation in the HCD cell. A deconvoluted $\mathrm{MS}^{2}$ spectrum shows detection of protein species that were targeted for fragmentation (Fig. 5a). HCD generated $b$ and $y$ ions from fragmentation at backbone amide positions that were mapped onto the sequence of the pMMO subunits (Fig. 5b), thereby localizing mass shifts ${ }^{38}$.

Notably, as shown in the graphical fragment maps in Fig. 5b, $\mathrm{PmoB}$ includes residues His 33 to Ile 414, consistent with the presence of a leader sequence that is cleaved posttranslationally ${ }^{36}$. PmoB residue Lys 36 was found to be methylated, a modification localized by the nTDMS to residues 33-51 and pinpointed by tandem MS of pepsin-digested peptides of 20Z-pMMO (Supplementary Fig. 6a). This lysine is located 8.3 $\AA$ from the $\mathrm{Cu}_{\mathrm{B}}$ site and does not appear methylated in the electron density map ${ }^{4}$, yet was present at $\sim 100 \%$ stoichiometry from the nTDMS data. It is not known whether this methylation is functionally important. PmoA was characterized to be Met $_{\mathrm{OFF}}$ and NtAc (Fig. 5b), as suggested by analysis of the micelle sample. The $\mathrm{MS}^{2}$ from the nanodisc reflects two populations of PmoC. A minor species is observed with $\mathrm{Met}_{\mathrm{OFF}}$ and NtAc while the major PmoC species is a truncated form without the first six N-terminal residues (MAATTE) (Fig. 5a, Supplementary Fig. 6b).

Localization of the copper-binding sites in pMMO. We next sought to determine the stoichiometry of metal binding to each pMMO subunit. The copper in 20Z-pMMO was determined by EPR analysis to be predominantly in the $\mathrm{Cu}(\mathrm{II})$ oxidation state ${ }^{4}$. Deconvolution of the $\mathrm{MS}^{2}$ generated upon ejection of pMMO from the nanodisc (Fig. 5a) revealed that the predominant PmoB proteoform has a mass consistent with a methylation $(14 \mathrm{Da})$ and one $\mathrm{Cu}(\mathrm{II})$ ion $(61.5 \mathrm{Da})$. Given that one copper ion remains associated with $\mathrm{PmoB}$ even after subunit ejection, we hypothesized that some copper might remain bound to fragments generated in the pseudo-MS ${ }^{3}$ analysis ${ }^{19}$ of this proteoform, thereby helping to verify the location of the $\mathrm{Cu}_{\mathrm{B}}$ site ${ }^{27}$. Two $b$-type fragment ions, $b_{165}$ and $b_{186}$ (corresponding to the numbering indicated in the graphical fragment map in Fig. 5b), were identified with mass shifts consistent with the binding of one $\mathrm{Cu}$ (II) ion (Supplementary Fig. 7) as demonstrated by the fitting of the theoretical isotopic distributions for copper-bound fragment ions. Notably, both copper-bound fragment ions occurred C-terminal to an aspartic acid residue, which is consistent with known 
a
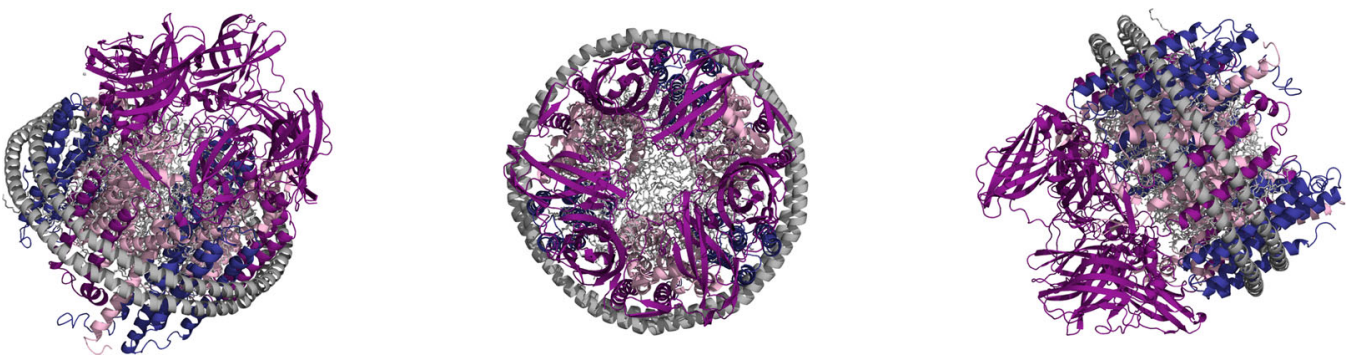

b

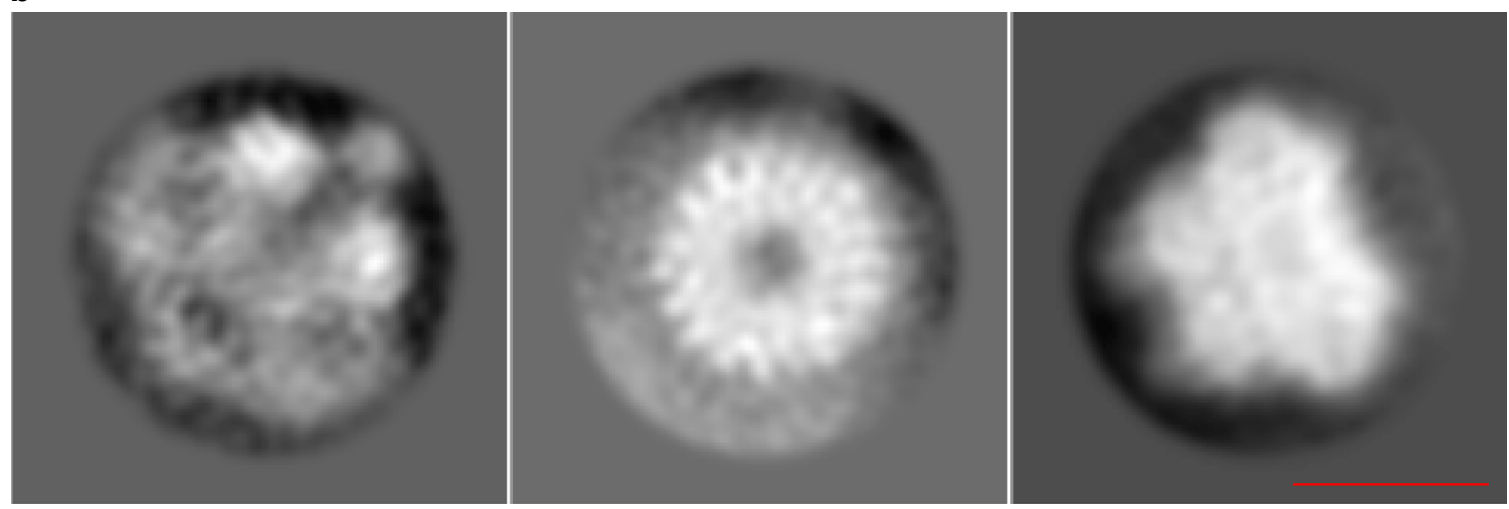

Fig. 4 Cryo-EM 2D class averages of 2OZ-pMMO in MSP2N2 nanodiscs. a A model of a pMMO nanodisc complex generated from the crystal structures of Methyloccocus capsulatus (Bath) pMMO (PDB accession code 3RGB) and MSP1D1 (from PDB accession code 6CC9). This model is for illustrative purposes and is not from 2D class averages. b Tilted, top, and side views of 2D class averages of 20Z-pMMO reconstituted in nanodiscs. A representative scale bar is shown as a $10 \mathrm{~nm}$ red line

fragmentation propensities under native $\mathrm{ESI}^{22}$. No copper binding was observed for fragment $b_{135}$ or for any other downstream $b$ ions. In congruence with the crystal structure, these copperbound fragment ions suggest that the copper-binding region (green underline in Supplementary Fig. 7) may be confined to the region spanning Trp 136-Asp 186, which contains the coordinating residues His 137 and His 139, but not the ligands to the bis-His site, His 48 and His 72. Copper binding fragment ions containing His 33 were not observed, perhaps due to the labile nature of this ligand suggested by the crystal structures ${ }^{3-6}$. Moreover, there is no copper binding observed in the C-terminal cupredoxin domain, previously suggested to bind $\sim 10$ copper ions ${ }^{39}$.

PmoC ejected from the pMMO-nanodisc complex has a proteoform present at $16 \%$ relative abundance that is consistent with a copper ion bound to the truncated PmoC species (Fig. 5a). Reduced copper stoichiometry in pMMO is observed upon nanodisc reconstitution (Supplementary Fig. 8), suggesting that bound copper may be lost from PmoC during the reconstitution. To determine whether the copper-binding stoichiometry of PmoC could be increased by exogenous addition of copper to the electrospray buffer, we added 1, 3, and 6 molar equivalents (eq.) of $\mathrm{Cu}(\mathrm{II})$ per protomer to $20 \mathrm{Z}$ pMMO in nanodiscs and analyzed these samples by nTDMS (Supplementary Fig. 9). We found that at 1 eq. of $\mathrm{Cu}(\mathrm{II})$ per protomer, $10.4 \%( \pm 3 \%)$ of $\mathrm{PmoC}$ is bound to copper. At 3 eq. of $\mathrm{Cu}(\mathrm{II})$, copper binding increases to a maximum of $27 \%( \pm 3 \%)$, with no further increase after addition of more copper. Notably, no additional copper binding is observed for $\mathrm{PmoB}$, as shown in the inset of Supplementary Fig. 9b. The precursor intensity of copper-bound PmoC proteoform was too low for these samples and thus was not fragmented successfully for metal localization. Taken together, the spectra generated from the micelle and nanodisc systems indicate that 20Z-pMMO binds one copper ion near the PmoB N-terminus and one copper ion in the PmoC subunit.

To validate the copper localization in 20Z-pMMO, pMMOs from other methanotrophs were investigated via nTDMS. Interestingly, PmoC copper binding was observed for samples of pMMO from $\mathrm{Mm}$. buryatense 5GB1C (5G-pMMO) in Triton $\mathrm{X}-100$ micelles. The $\mathrm{MS}^{1}$ analysis of $5 \mathrm{G}-\mathrm{pMMO}$ shows a predominant mass species that correlates to a $\mathrm{pMMO}$ protomer bound to two copper ions (Supplementary Figs. 10, 11a), similar to 20Z-pMMO in micelles. MS $^{2}$ ejection (Supplementary Figs. 11b, 12) of the subunits confirms that the predominant species of PmoB also contains one copper ion and has Lys 36 methylation. While $\mathrm{MS}^{2}$ of 5G-pMMO in micelles indicated copper binding to the PmoC subunit, this was not the case for the 20Z-pMMO PmoC in micelles, possibly due to structural differences between the two PmoC subunits. Unfortunately, 5GpMMO in nanodiscs exhibited poor ejection from the nanodisc complex and could not be characterized via nTDMS.

We then investigated pMMO from Methylocystis sp. strain Rockwell (Rockwell-pMMO). In the crystal structure of RockwellpMMO, the PmoC site is occupied by copper ${ }^{3}$. Unlike 5GpMMO, Rockwell-pMMO in MSP1E3D1 nanodiscs (Supplementary Fig. 13) ejected well from the nanodisc complex and was thus amenable to nTDMS analysis of the subunits. Subunit ejection (Supplementary Fig. 14, Fig. 6a) and subsequent fragmentation of Rockwell-pMMO led to the identification of the PmoB and PmoC subunits (Fig. 6c). Similar to 20Z-pMMO, PmoB showed an intact mass shift of $61.5 \mathrm{Da}$ that suggests $100 \%$ occupancy of a single $\mathrm{Cu}(\mathrm{II})$ ion. Unlike 20Z- and 5G-pMMO, PmoB methylation was not detected, indicating that this PTM may not be necessary for activity and may be specific to Methylomicrobium pMMOs. Truncated PmoC was observed in the apo form, but close examination of the spectrum (Fig. 6a) reveals a low abundance peak that is shifted by the mass of copper, consistent 


\section{a Deconvoluted MS²: 20Z-pMMO subunits ejected from nanodisc-pMMO complex}

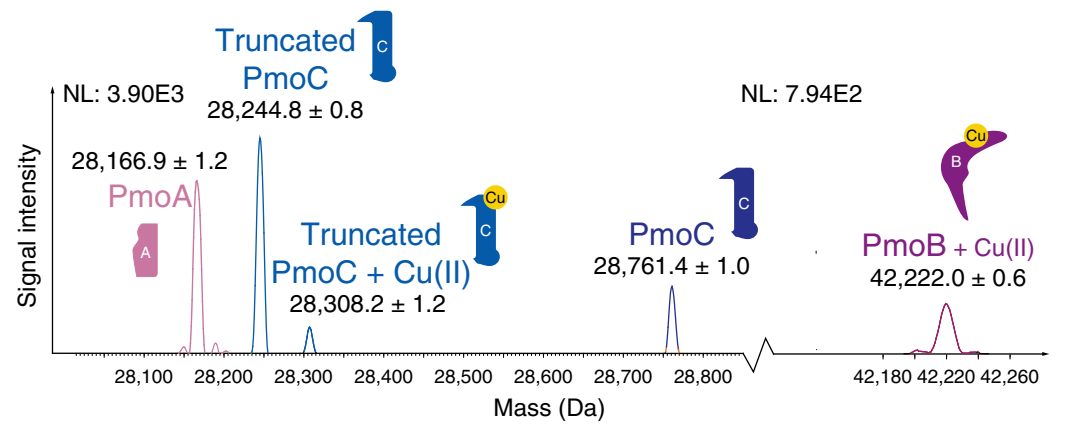

20Z-pMMO theoretical MW accounting for PTMs:

\begin{tabular}{|c|c|}
\hline PmoA & $\begin{array}{l}28,167.1 \mathrm{Da} \\
\Delta \mathrm{m}: 0.2 \mathrm{Da}\end{array}$ \\
\hline $\mathrm{PmoB}+\mathrm{Cu}(\mathrm{II})$ & $\begin{array}{l}42,222.7 \mathrm{Da} \\
\Delta \mathrm{m}: 0.7 \mathrm{Da}\end{array}$ \\
\hline PmoC & $\begin{array}{l}28,762.3 \mathrm{Da} \\
\Delta \mathrm{m}: 1.1 \mathrm{Da}\end{array}$ \\
\hline $\begin{array}{r}\text { Truncated } \\
\text { PmoC }\end{array}$ & $\begin{array}{l}28,246.8 \mathrm{Da} \\
\Delta \mathrm{m}: 2.0 \mathrm{Da}\end{array}$ \\
\hline $\begin{array}{r}\text { Truncated } \\
\mathrm{PmoC}+\mathrm{Cu}(\mathrm{II})\end{array}$ & $\begin{array}{l}28,308.3 \mathrm{Da} \\
\Delta \mathrm{m}: 0.1 \mathrm{Da}\end{array}$ \\
\hline
\end{tabular}

\section{b MS ${ }^{3}$ : Fragmentation of ejected 20Z-pMMO subunits}

PmoA $\quad p$-score: $3.2 \times 10^{-39}$

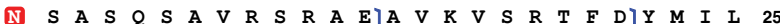

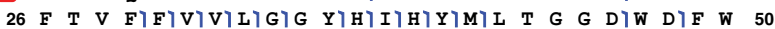

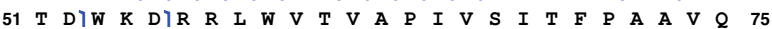
$\begin{array}{lllllllllllllllllllllllllll}76 & \text { A } & V & \text { L } & \text { W } & \text { W } & R & Y & R & \text { I } & A & \text { W } & G & A & T & \text { L } & C & \text { V } & \text { L } & G & \text { L } & \text { L } & \text { L } & G & E & \text { W } & 100\end{array}$

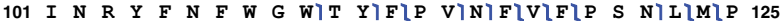
126 G ALILV L D D V I IL M LIS N S M T L L T A VIVLG G L A 150 151 LW LG L L

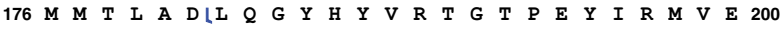

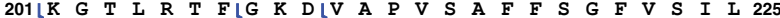

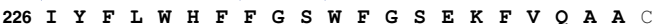

\section{PmoC $\quad p$-score: $3.7 \times 10^{-14}$}

太 $\begin{array}{lllllllllllllllllllllllllll}26 & A & S & L & Y & \text { L } & V & F & Y & A & \text { W } & \text { V } & R & \text { W } & \text { Y } & \text { E } & G & \text { V } & \text { Y } & G & \text { W } & S & A & G & \text { L } & \text { Dl } & 50\end{array}$ $\begin{array}{lllllllllllllllllllllllllll}51 & S & F & A & P & E & F & E & T & Y & W & M & N & F & L & Y & I & E & M & V & L & E & V & L & T & A & 75\end{array}$ $\begin{array}{lllllllllllllllllllllllllll}76 & S & V & L & W & G & Y & I & W & K & S & R & D & R & K & V & M & S & I & T & P & R & E & E & L & R & 100\end{array}$ $\begin{array}{lllllllllllllllllllllllllll}101 & R & H & F & T & H & \text { W } & T & \text { W } & \text { L } & \text { M } & \text { M } & \text { Y } & G & \text { I } & \text { A } & \text { I } & \text { Y } & \text { F } & G & \text { A } & \text { S } & \text { Y } & F & \text { T } & \text { E } & 125\end{array}$ $\begin{array}{lllllllllllllllllllllllllll}126 & O & D & G & T & W & H & O & T & I & V & R & D & T & D & F & T & P & S & H & I & I & E & F & Y & L & 150\end{array}$ $\begin{array}{lllllllllllllllllllllllllll}151 & S & Y & P & I & Y & I & I & T & G & G & A & S & F & L & Y & A & K & T & R & L & P & T & Y & Q & Q & 175\end{array}$ 176 G L S L $Q$ Y L VIVIVLVLGLP F MLILLLP N VLG L N $\quad$ E W 200

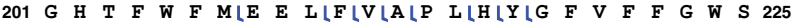
226 A LLG VLLLG VLILN ILELL G ALL $S$ K

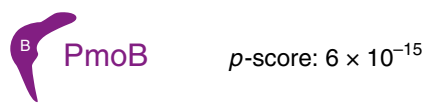

N 33 H G E R S Q A A F M R M R T I H W F D D L N]W]S KןDl 57 58 O V S V V N ElT M S I S G K F H VIF AlG WlP ElT V DI 82

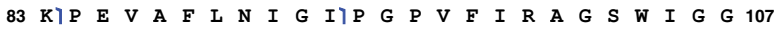
$\begin{array}{lllllllllllllllllllllllllll}108 & Q & L & V & P & R & S & V & S & L & E & L & G & E & \text { T } & \text { Y } & \text { E } & \text { F } & K & V & \text { L } & \text { L } & K & \text { A } & R & R & 132\end{array}$ 133 P G G D JW $\begin{array}{lllllllllllllllllllllllll}158 & T & I & T & G & S & M & G & D & F & K & N & P & I & T & T & L & T & G & E & T & I & D & L & E\end{array}$ $\begin{array}{lllllllllllllllllllllllll}183 & \text { Y } & \text { A } & \text { L } & \text { D I G } & \text { V } & \text { Y } & \text { G } & \text { W } & \text { H } & \text { L } & \text { F } & \text { W } & \text { Y } & \text { L } & \text { L } & \text { G } & \text { V } & \text { A } & \text { W } & \text { M } & \text { V } & \text { Y } & \text { WlC } & 207\end{array}$ $\begin{array}{lllllllllllllllllllllllllll}208 & R & K & P & V & F & I & P & R & R & I & A & V & D & A & G & K & A & D & S & L & I & T & P & T & D & 232\end{array}$

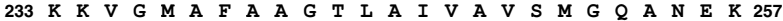
$\begin{array}{lllllllllllllllllllllllllll}258 & \text { Y } & P & V & T & T & P & \text { L } & \text { Q } & \text { A } & G & \text { L } & M & R & G & \text { I } & K & S & \text { L } & E & \text { L } & P & Q & P & T & V & 282\end{array}$ $\begin{array}{lllllllllllllllllllllllllll}283 & S & V & K & V & V & D & A & S & Y & R & V & P & G & R & A & M & Q & M & T & L & E & I & T & N & N & 307\end{array}$ $\begin{array}{llllllllllllllllllllllllllll}308 & G & D & S & A & V & R & L & A & E & F & N & T & A & S & V & R & F & L & D & A & D & V & Y & E & D & 332\end{array}$

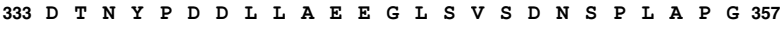
$\begin{array}{lllllllllllllllllllllllllll}358 & \text { E } & T & R & T & V & D & V & T & \text { A } & S & D & \text { A } & \text { A } & \text { W } & \text { E } & \text { V } & \text { Y } & R & \text { L } & \text { A } & \text { D L L } & \text { I } & Y & \text { D } & 382\end{array}$

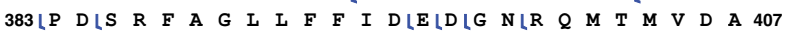
$\begin{array}{llllllllll}408 & \text { P } & \text { L } & \text { I } & P & T & F & \text { I } & \text { C }\end{array}$

N-terminal fragment

L C-terminal fragment

乙 Bidirectional ( $\mathrm{N}$ and $\mathrm{C}$ ) fragment
N N-terminal acetylation

$\mathrm{K}$ Lysine methylation

Fig. 5 nTDMS analysis of 20Z-pMMO in MSP2N2 nanodiscs. a Deconvoluted MS² showing detection of 20Z-pMMO subunit masses upon ejection from the nanodisc-pMMO complex. The panel on the right contains the theoretical masses of the subunits, accounting for the modifications characterized by tandem MS. b Graphical fragment maps of pMMO subunits derived from the $\mathrm{MS}^{3}$ step in the nTDMS platform. Fragments are depicted as blue flags in the graphical fragment maps, indicating which regions of the protein sequence can be accounted for in mass by the fragments. The pMMO subunits were found to be modified by $\mathrm{N}$-terminal acetylation ( $\mathrm{PmoA}$ and $\mathrm{PmoC}$ ), $\mathrm{N}$-terminal truncation $(\mathrm{PmoC}$ ), and lysine methylation (PmoB). The graphical fragment map of $\mathrm{PmoB}$ begins at residue His 33, showing cleavage of the known $\mathrm{N}$-terminal signal peptide

with inductively coupled plasma mass spectrometry (ICP-MS) data (vide infra) and with a labile metal interaction that is partially lost at high collision voltages or during nanodisc reconstitution, as observed for 20Z-pMMO.

Linking pMMO activity to copper binding by PmoC. In the crystal structures, the PmoC metal binding site is occupied by zinc $^{3,5-7}$ or copper (Rockwell-pMMO) ${ }^{3}$, or is completely disordered ${ }^{4}$. However, it has thus far been unclear whether metal binding at this site is functionally relevant ${ }^{3}$. Given that the nTDMS data indicate the presence of some copper in the PmoC subunits from three different organisms, and that pMMO activity requires more than one copper ion, we investigated the effect of exogenous copper addition on Rockwell-pMMO by both nTDMS and activity assays. Membrane-bound Rockwell-pMMO exhibits the highest methane oxidation activity at $30^{\circ} \mathrm{C}$ out of all characterized pMMOs $^{3,4}$, but upon reconstitution into nanodiscs, the activity dramatically decreased compared to that of the as-isolated membranes (Fig. 7a).
Upon addition of one equivalent of $\mathrm{CuSO}_{4}$ per pMMO protomer during the nanodisc reconstitution process, the methane oxidation activity of the pMMO-nanodisc complex increased six-fold (Fig. 7a). The activity was higher than that of membrane-bound pMMO using duroquinol as a reductant, similar to the increased activity observed for pMMOs reconstituted into bicelles ${ }^{4}$. ICP-MS analysis of the pMMO-nanodisc samples prepared with and without copper supplementation showed that in the absence of copper supplementation, $0.7 \pm 0.2$ $(n=4)$ copper ion per pMMO protomer is present (Fig. 7b). Upon copper addition and incorporation into the pMMOnanodisc complex, the copper content increased to $1.8 \pm 0.5(n=$ 5) copper ions per $\mathrm{pMMO}$ protomer, consistent with the presence of up to two copper-binding sites. These copper-supplemented Rockwell-pMMO nanodisc samples were then analyzed by nTDMS to determine the location of the second copper ion (Fig. 6b, Supplementary Fig. 15). The nTDMS analysis of the active Rockwell-pMMO prepared with exogenous addition of copper showed a significantly higher amount of copper bound PmoC (37 $\pm 2 \%$, Fig. 6a) compared to the inactive sample 
a Deconvoluted MS²: Rockwell-pMMO subunits ejected from nanodiscs

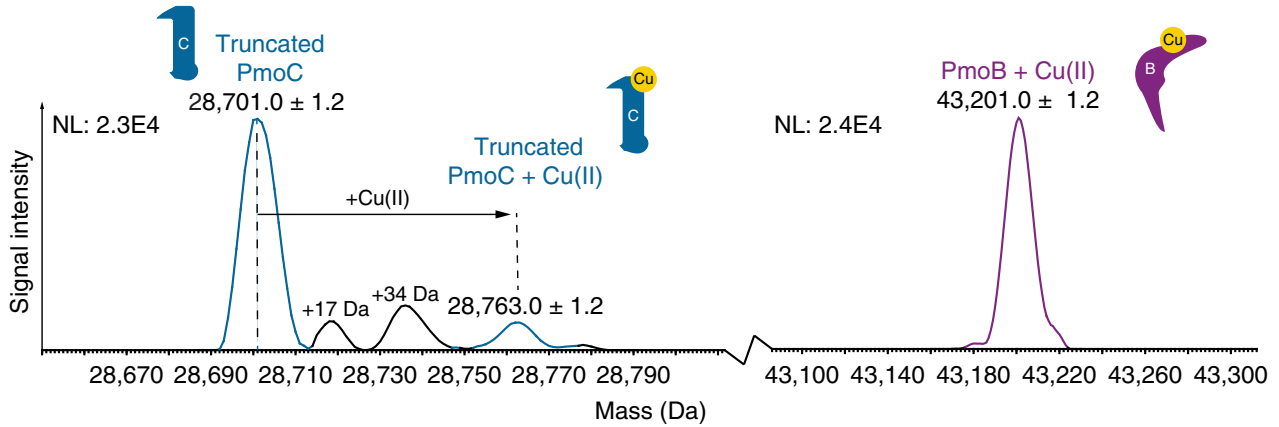

b

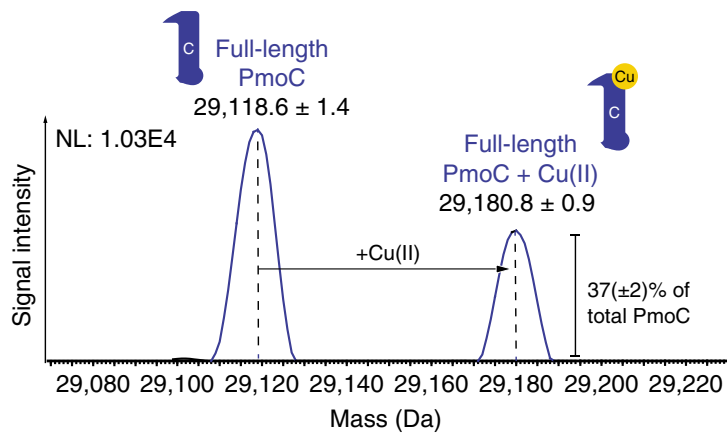

Rockwell-pMMO theoretical MW accounting for PTMs:

\begin{tabular}{|rl|}
\hline PmoB + Cu(II) & $43,201.7 \mathrm{Da}$ \\
& $\Delta \mathrm{m}: 0.7 \mathrm{Da}$ \\
Truncated & $28,702.6 \mathrm{Da}$ \\
PmoC & $\Delta \mathrm{m}: 1.6 \mathrm{Da}$ \\
Truncated & $28,764.1 \mathrm{Da}$ \\
PmoC + Cu(II) & $\Delta \mathrm{m}: 1.1 \mathrm{Da}$ \\
\hline
\end{tabular}

\section{MS $^{3}$ : Fragmentation of ejected Rockwell-pMMO subunits}
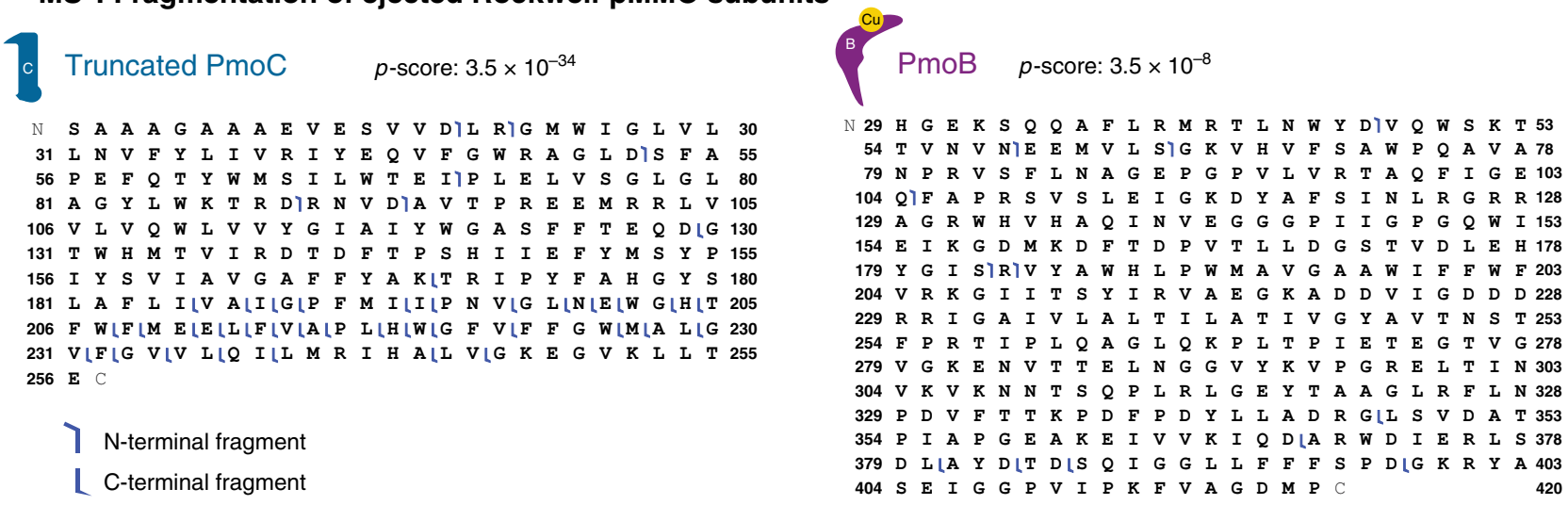

Fig. 6 nTDMS analysis of Rockwell-pMMO in MSP1E3D1 nanodiscs. a Deconvoluted MS² demonstrating detection of Rockwell-pMMO subunit masses upon ejection from the nanodisc without or $\mathbf{b}$ with copper supplementation during nanodisc reconstitution. The panel on the right contains the theoretical masses of the subunits, accounting for the modifications characterized by tandem MS. The two species shifted in mass from PmoC by +17 and +34 Da likely correspond to the replacement of one and two protons by ammonium adducts (Th. 17.03 Da and 34.06 Da, respectively) on PmoC commonly observed in $\mathrm{nESI}^{65}$. c The $\mathrm{MS}^{3}$ of truncated $\mathrm{PmoC}$ and $\mathrm{PmoB}$ yielded fragment ions, depicted as blue flags in the graphical fragment maps that indicate which regions of the protein sequence can be accounted for in mass by the fragments. Truncated PmoC lacks the first five residues of the $\mathrm{N}$-terminus (MSSTT), and its graphical fragment map begins at residue Ser 6. The graphical fragment map of PmoB begins at residue His 29, consistent with the cleavage of the known N-terminal signal peptide

(Fig. 6a). The correlation between this result and the activity data suggests that the copper ion in PmoC, likely corresponding to the spectroscopically assigned $\mathrm{Cu}_{\mathrm{C}}$ site ${ }^{14}$, is essential for methane oxidation activity.

\section{Discussion}

The nTDMS analysis presented here advances our understanding of pMMO in several ways. First, we have obtained accurate intact masses for the pMMO protomer and individual subunits and have detected a previously unknown methylation of residue Lys 36 in the PmoB subunits of 20Z-pMMO and 5G-pMMO. The importance of this PTM, which is not found in Rockwell-pMMO, is unclear. This methylation might afford protection against reaction with radicals and oxidative damage, as proposed for methyl-coenzyme $M$ reductase ${ }^{40}$ and lytic polysaccharide monooxygenases ${ }^{41}$, or provide additional hydrophobic interactions important for methane binding. Alternatively, this methylated lysine may be an off-target product formed by methyltransferases responsible for synthesizing osmolytes in haloalkaliphiles ${ }^{42}$. The observation of these modifications, including the truncated proteoforms of PmoC, highlights the importance of measuring the intact masses of proteoforms, as such modifications were not readily observed in previous biochemical studies ${ }^{4}$ and may have functional implications. 

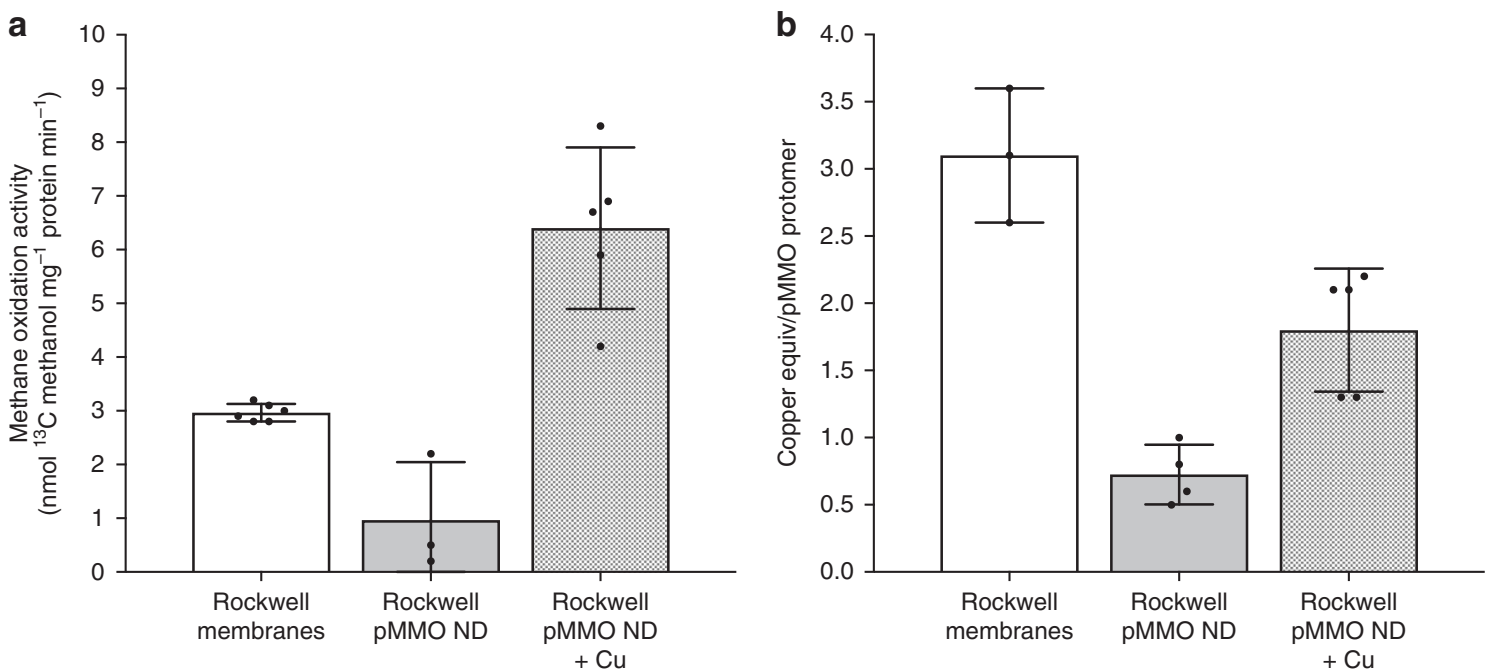

Fig. 7 Activity and metal content of Rockwell-pMMO in nanodiscs. a ${ }^{13} \mathrm{C}$ methane oxidation activity of Rockwell-pMMO in membranes and in nanodiscs (ND) without or with copper supplementation. Values are shown in $\mathrm{nmol}^{13} \mathrm{C}$ methanolømg ${ }^{-1}$ protein $\bullet \mathrm{min}^{-1}$. Error bars represent standard deviation of individual measurements (black dots) of $n=6,3$, and 5, respectively. b Copper equivalents per pMMO protomer for Rockwell-pMMO in membranes and in nanodiscs without or with copper supplementation. Error bars represent standard deviation of individual measurements (black dots) of $n=3,4$, and 5 , respectively. Source data are provided as a Source Data file

Second, we have localized the copper sites in pMMO and have established a correlation between the PmoC site and methane oxidation activity. The observation of one copper ion in $\mathrm{PmoB}$ and one copper ion in PmoC is consistent with in vivo EPR and DEER data ${ }^{14}$, and confirms that the two copper ions detected in pMMO by metal analysis occupy these two sites. Previous studies have suggested that the PmoC site is important for activity. In particular, mutagenesis of the $\mathrm{PmoC}$ metal binding ligands in hydrocarbon monooxygenase (HMO), a homolog of pMMO, abrogates enzyme activity ${ }^{43}$. Additionally, inhibition of pMMO by zinc has been attributed to zinc binding in the PmoC site 3 . However, the different occupancies of this site in the crystal structures, including the presence of $\mathrm{zinc}^{3,5-7}, \operatorname{copper}^{3}$, or no observable metal ion ${ }^{4}$, have obscured its biological relevance. Here we show that copper and activity loss upon nanodisc reconstitution can be restored by addition of exogenous copper, which increases copper occupancy of the PmoC site (Figs 6, 7). These results suggest that the $\mathrm{PmoC} \mathrm{Cu}_{\mathrm{C}}$ site plays an important role in methane oxidation. Disruption of the native membrane may account for the increased lability of this site as compared to the consistently observed $\mathrm{Cu}_{\mathrm{B}}$ site, which is in the periplasmic region of PmoB.

The $\mathrm{Cu}_{\mathrm{C}}$ site and the respective roles of both monocopper sites in pMMO must be investigated further. The $\mathrm{Cu}_{\mathrm{C}}$ site resides in a disordered yet highly conserved region of PmoC, and additional information on its coordination environment is desirable. It may be that the two monocopper centers function analogously to the non-coupled monocopper sites in peptidylglycine $\alpha$-hydroxylating monooxygenase and dopamine $\beta$ monooxygenase ${ }^{44}$, in which the $\mathrm{Cu}_{\mathrm{M}}$ site is involved in $\mathrm{O}_{2}$ activation and the $\mathrm{Cu}_{\mathrm{H}}$ site, located $11 \AA$ away across a solventfilled cleft, provides the second electron to the $\mathrm{Cu}_{\mathrm{M}}$ site for turnover. In the pMMO crystal structures, the PmoB and PmoC metal centers are $20 \AA$ apart, but in the membrane, the PmoB periplasmic domain may shift towards the lipid bilayer, altering this distance. The combined activity and nTDMS data show that increased loading of the $\mathrm{Cu}_{\mathrm{C}}$ site enhances activity, consistent with a recent proposal that methane and oxygen bind at this site ${ }^{14}$. It remains unclear whether the $\mathrm{Cu}_{\mathrm{B}}$ site is absolutely necessary for activity. While this site is consistently observed, its three histidine ligands are not present in the verrucomicrobial pMMO PmoB sequences ${ }^{45}$, suggesting that methane oxidation may occur in its absence. In comparison, the $\mathrm{Cu}_{\mathrm{C}}$ ligands are conserved in all $\mathrm{pMMOs}^{45,46}$, and its location in the intracytoplasmic membrane may facilitate methane access ${ }^{47}$.

It is also important to note that the activity of the RockwellpMMO nanodisc complex (Fig. 7a) is significantly lower than that of whole cells ${ }^{1,3}$, which could result from loss of additional copper sites prior to nanodisc reconstitution and nTDMS analysis. However, recent spectroscopic analysis indicates that the $\mathrm{Cu}_{\mathrm{B}}$ and $\mathrm{Cu}_{\mathrm{C}}$ sites are also present in whole cells ${ }^{14}$, suggesting that the activity loss is primarily due to other factors, such as loss of potential interacting partners ${ }^{48}$, use of a nonphysiological reductant ${ }^{49}$, or disruption of the membrane structure and composition ${ }^{4,50}$. These considerations may differentially affect the two monocopper sites.

Through an alternative approach coupling membrane mimetics with nTDMS analysis, we have gained valuable insights into the active form of pMMO. The nTDMS platform relies on the isolation of distinct mass peaks using a quadrupole mass filter optimized for selection of high $\mathrm{m} / \mathrm{z}$ ions produced by native electrospray ${ }^{19}$. Native-mode TDMS also circumvents protein contamination issues, is sensitive to the dynamic nature of large protein assemblies, and can resolve functionally different proteoforms of metal-bound subunits. The technological advances described here can overcome difficulties intrinsic to membrane proteins $^{51}$ and challenges associated with nanodisc samples ${ }^{29,31}$. Moreover, the ability to localize metal ions fills a gap in the traditional bioinorganic toolbox to characterize metalloproteins. Therefore, the experimental findings not only impact pMMO, but have broad implications for the improved characterization of myriad challenging membrane-bound complexes in metallobiology.

\section{Methods}

Membrane scaffold protein expression and purification. The membrane scaffold proteins MSP1E3D1 and MSP2N2, each with TEV-cleavable N-terminal 7histidine tags, were expressed and purified ${ }^{32}$. E. coli BL21(DE3) cells were transformed with plasmids pMSP1E3D1 (Addgene, \#20066) or pMSP2N2 (Addgene, \#29520), and $1 \mathrm{~L}$ cultures were inoculated at a starting $\mathrm{OD}_{600}$ of 0.1 in Terrific Broth media and grown for $4 \mathrm{~h}$ at $37^{\circ} \mathrm{C}$ with shaking at $200 \mathrm{rpm}$ until an $\mathrm{OD}_{600}$ of 
2.3 was reached. The cultures were induced with $1 \mathrm{mM}$ isopropyl $\mathrm{B}-\mathrm{D}-1$ thioglactopyranoside (IPTG) (RPI) and were grown for an additional $4 \mathrm{~h}$ at $37^{\circ} \mathrm{C}$ for protein expression, yielding $5 \mathrm{~g}$ wet cell pellets per $\mathrm{L}$ of culture. Cell pellets were harvested by centrifugation at $8000 \times \mathrm{g}$ for $10 \mathrm{~min}$, flash-frozen in liquid nitrogen, and stored at $-80^{\circ} \mathrm{C}$.

Cells were resuspended in buffer A ( $40 \mathrm{mM}$ Tris, pH 7.3, $250 \mathrm{mM} \mathrm{NaCl}, 20 \mathrm{mM}$ imidazole) at $50 \mathrm{~mL}$ per $10 \mathrm{~g}$ cell pellet. EDTA-free protease inhibitor cocktail (1 tablet per $50 \mathrm{~mL}$, Roche) and chicken egg lysozyme $(10 \mathrm{mg}$ per $50 \mathrm{~mL}$, SigmaAldrich) were added. Once resuspended, Triton X-100 (Sigma-Aldrich) was added to a final concentration of $1 \% \mathrm{v} / \mathrm{v}$ and stirred. Cells were lysed on ice by sonication at $35 \%$ amplitude for $10 \mathrm{~min}$ ( $1 \mathrm{~s}$ on, $1 \mathrm{~s}$ off) and cell debris was removed by centrifugation at $10,000 \times \mathrm{g}$ for $30 \mathrm{~min}$. The soluble fraction was loaded onto a column containing $10 \mathrm{~mL}$ Ni-NTA beads (Qiagen). The beads were washed with $50 \mathrm{~mL}$ of buffer B ( $40 \mathrm{mM}$, Tris pH 7.3, $250 \mathrm{mM} \mathrm{NaCl}, 20 \mathrm{mM}$ imidazole, $50 \mathrm{mM}$ sodium cholate) followed by $150 \mathrm{~mL}$ buffer $\mathrm{A}$. The protein was then eluted with buffer C ( $40 \mathrm{mM}$ Tris, pH 7.3, $250 \mathrm{mM} \mathrm{NaCl}, 250 \mathrm{mM}$ imidazole).

The elution fractions were pooled and TEV protease was added at a mass ratio $\mathrm{w} / \mathrm{w}$ of 1:40 TEV:protein. This mixture was dialyzed using a 10 MWCO SnakeSkin dialysis tubing (Thermo Fisher Scientific) in $1 \mathrm{~L}$ of buffer D ( $40 \mathrm{mM}$ Tris, pH 7.3, $250 \mathrm{mM} \mathrm{NaCl}, 20 \mathrm{mM}$ imidazole, $1 \mathrm{mM}$ EDTA) overnight with a buffer change after $1 \mathrm{~h}$. TEV protease was removed by applying the sample to a Ni-NTA column and collecting the flowthrough, which was then dialyzed overnight against $1 \mathrm{~L}$ of buffer E (25 mM PIPES, pH 7.3, $250 \mathrm{mM} \mathrm{NaCl}$ ) with a buffer change after $1 \mathrm{hr}$. The purified MSP proteins were concentrated using an Amicon centrifugal concentrator (10 kDa MWCO, Millipore) to a concentration of $4.5 \mathrm{mg} / \mathrm{mL}$, measured by $A_{280}$ using extinction coefficients of 26,930 and $36,900 \mathrm{M}^{-1} \cdot \mathrm{cm}^{-1}$ for MSP1E3D1 and MSP2N2, respectively. The protein was flash frozen on liquid nitrogen and stored at $-80{ }^{\circ} \mathrm{C}$.

Methanotroph cell growth. Mm. alcaliphilum $20 \mathrm{Z}$ and Mm. buryatense $5 \mathrm{~GB} 1 \mathrm{C}$ were cultured following established methods ${ }^{4,52}$. Briefly, $12 \mathrm{~L}$ bioreactor cultures were grown in $1 \mathrm{X}$ modified nitrate mineral salts (NMSA) medium, $0.5 \mathrm{M} \mathrm{NaCl}$ (Mm. alcaliphilum 20Z) or $0.130 \mathrm{M} \mathrm{NaCl}(\mathrm{Mm}$. buryatense $5 \mathrm{~GB} 1 \mathrm{C}), 2.3 \mathrm{mM}$ phosphate buffer, $50 \mathrm{mM}$ carbonate buffer, $\mathrm{pH} 9.5$, supplemented with $40 \mu \mathrm{M}$ $\mathrm{CuSO}_{4} \cdot 5 \mathrm{H}_{2} \mathrm{O}$ and trace elements solution ( $\mathrm{Mm}$. alcaliphilum $20 \mathrm{Z} 2000 \mathrm{X}$ stock solution: $0.5 \mathrm{~g} / \mathrm{L} \mathrm{Na}_{2} \bullet \mathrm{ETDA}, 0.2 \mathrm{~g} / \mathrm{L} \mathrm{FeSO}_{4} \bullet 7 \mathrm{H}_{2} \mathrm{O}, 0.01 \mathrm{~g} / \mathrm{L} \mathrm{ZnSO}_{4} \cdot 7 \mathrm{H}_{2} \mathrm{O}, 0.003 \mathrm{~g} / \mathrm{L}$ $\mathrm{MnCl}_{2} \bullet 4 \mathrm{H}_{2} \mathrm{O}, 0.03 \mathrm{~g} / \mathrm{L} \mathrm{H}_{3} \mathrm{BO}_{3}, 0.02 \mathrm{~g} / \mathrm{L} \mathrm{CoCl}_{2} \bullet 6 \mathrm{H}_{2} \mathrm{O}, 0.002 \mathrm{~g} / \mathrm{L} \mathrm{NiCl}_{2} \bullet 6 \mathrm{H}_{2} \mathrm{O}, 0.003$ $\mathrm{g} / \mathrm{L} \mathrm{Na}_{2} \mathrm{MoO}_{4} \bullet 2 \mathrm{H}_{2} \mathrm{O} ; \mathrm{Mm}$. buryatense $5 \mathrm{~GB} 1 \mathrm{C} 500 \mathrm{x}$ stock solution: $1.0 \mathrm{~g} / \mathrm{L}$ $\mathrm{Na}_{2} \bullet$ EDTA, $2.0 \mathrm{~g} / \mathrm{L} \mathrm{FeSO}_{4} \bullet 7 \mathrm{H}_{2} \mathrm{O}, 0.8 \mathrm{~g} / \mathrm{L} \mathrm{ZnSO}_{4} \bullet 7 \mathrm{H}_{2} \mathrm{O}, 0.03 \mathrm{~g} / \mathrm{L} \mathrm{MnCl}_{2} \bullet 4 \mathrm{H}_{2} \mathrm{O}$ $0.03 \mathrm{~g} / \mathrm{L} \mathrm{H}_{3} \mathrm{BO}_{3}, 0.2 \mathrm{~g} / \mathrm{L} \mathrm{CoCl}_{2} \bullet 6 \mathrm{H}_{2} \mathrm{O}, 0.02 \mathrm{~g} / \mathrm{L} \mathrm{NiCl}_{2} \bullet 6 \mathrm{H}_{2} \mathrm{O}, 0.05 \mathrm{~g} / \mathrm{L}$ $\mathrm{Na}_{2} \mathrm{MoO}_{4} \cdot 2 \mathrm{H}_{2} \mathrm{O}$ ). Mc. sp. str. Rockwell cells were cultured in 1X NMS medium, $3.9 \mathrm{mM}$ phosphate buffer, $\mathrm{pH} 6.8$, supplemented with $50 \mu \mathrm{M} \mathrm{CuSO}_{4} \cdot 5 \mathrm{H}_{2} \mathrm{O}, 40 \mu \mathrm{M}$ $\mathrm{FeSO}_{4} \cdot 7 \mathrm{H}_{2} \mathrm{O}$, and trace elements solution (500X stock solution: $0.288 \mathrm{~g} / \mathrm{L}$ $\mathrm{ZnSO}_{4} \cdot 7 \mathrm{H}_{2} \mathrm{O}, 0.166 \mathrm{~g} / \mathrm{L} \mathrm{MnCl}_{2} \bullet 4 \mathrm{H}_{2} \mathrm{O}, 0.062 \mathrm{~g} / \mathrm{L} \mathrm{H}_{3} \mathrm{BO}_{3}, 0.048 \mathrm{~g} / \mathrm{L}$

$\left.\mathrm{Na}_{2} \mathrm{MoO}_{4} \bullet 2 \mathrm{H}_{2} \mathrm{O}, 0.048 \mathrm{~g} / \mathrm{L} \mathrm{CoCl}_{2} \bullet 6 \mathrm{H}_{2} \mathrm{O}, 0.083 \mathrm{~g} / \mathrm{L} \mathrm{KI}\right)^{3}$. These cultures were grown under a continuous gas flow using a 1:3 methane-to-air ratio at $1.2 \mathrm{~L} / \mathrm{min}$ at $30{ }^{\circ} \mathrm{C}$ with $300 \mathrm{rpm}$ agitation. All bioreactor cultures were harvested at an $\mathrm{OD}_{600}$ of $8-10$, centrifuged at $8000 \times \mathrm{g}$ for $1 \mathrm{~h}$, flash frozen in liquid nitrogen, and stored at $-80^{\circ} \mathrm{C}$

Membrane isolation. Membranes from the three methanotrophs were isolated following established methods ${ }^{3,4,52}$. Ten grams of cells were resuspended in $100 \mathrm{~mL}$ of $25 \mathrm{mM}$ PIPES, pH 7.3, $500 \mathrm{mM} \mathrm{NaCl}$ (Mm. alcaliphilum 20Z) or $250 \mathrm{mM} \mathrm{NaCl}$ (Mm. buryatense $5 \mathrm{GB1C})$, supplemented with EDTA-free protease inhibitor tablets (Roche). Resuspended cells were sonicated for $1.5 \mathrm{~min}$ with an $1 \mathrm{~s}$ on, $3 \mathrm{~s}$ off interval at $25 \%$ sonication amplitude. Mc. sp. str. Rockwell cells ( $16 \mathrm{~g})$ were resuspended in $70 \mathrm{~mL}$ of $25 \mathrm{mM}$ PIPES, pH 7.3, $250 \mathrm{mM} \mathrm{NaCl}$, supplemented with $500 \mu \mathrm{M} \mathrm{CuSO}_{4} \cdot 5 \mathrm{H}_{2} \mathrm{O}$, and sonicated for $7 \mathrm{~min}$ with an $1 \mathrm{~s}$ on, $3 \mathrm{~s}$ off interval at $25 \%$ sonication amplitude. All lysed cells were centrifuged at $8000 \times g$ for $1 \mathrm{~h}$ at $4{ }^{\circ} \mathrm{C}$. The supernatant was then centrifuged at $100,000 \times g$ for $1 \mathrm{~h}$ at $4^{\circ} \mathrm{C}$. The resulting membrane pellet was washed twice in a Dounce homogenizer with $25 \mathrm{mM}$ PIPES, $\mathrm{pH}$ 7.3., $250 \mathrm{mM} \mathrm{NaCl} .1 \mathrm{~mL}$ aliquots of membranes $(5-10 \mathrm{mg} / \mathrm{mL})$ were flash frozen in liquid nitrogen and stored at $-80^{\circ} \mathrm{C}$. Protein concentrations were measured using the DC Lowry Assay (Bio-Rad) with BSA as a standard.

pMMO solubilization. pMMO was solubilized from the membranes using $1.2 \mathrm{mg}$ of n-Dodecyl $\beta$-D-maltoside (DDM) (Anatrace) per $1 \mathrm{mg}$ of protein at $4^{\circ} \mathrm{C}$ for 1 $\mathrm{h}^{3,4,52}$. Membranes were pelleted at $100,000 \times \mathrm{g}$ for $30 \mathrm{~min}$ at $4^{\circ} \mathrm{C}$, and the solubilized protein fraction was collected and buffer exchanged into $25 \mathrm{mM}$ PIPES, $\mathrm{pH}$ $7.3,250 \mathrm{mM} \mathrm{NaCl}, 0.02 \%$ DDM using a $100 \mathrm{kDa}$ MWCO Amicon centrifugal concentrator (Millipore). Protein concentrations were measured using the DC Lowry Assay (Bio-Rad) with BSA as a standard.

pMMO reconstitution into nanodiscs using dialysis. 20Z- and Rockwell-pMMO were reconstituted into nanodiscs via dialysis using the membrane scaffold proteins MSP1E3D1 or MSP2N2 and 1-palmitoyl-2-oleoyl-sn-glycero-3-phosphocholine (POPC) lipids. A stock of $50 \mathrm{mM}$ POPC was prepared from POPC powder (Avanti) in $100 \mathrm{mM}$ sodium cholate, $25 \mathrm{mM}$ PIPES, pH 7.3 and $250 \mathrm{mM} \mathrm{NaCl}$. POPC was dissolved into the buffer by cycling the glass tube through $20 \mathrm{~s}$ of vortexing, a $20 \mathrm{~s}$ sonication step in an ultrasonic bath, and a $20 \mathrm{~s}$ incubation at $50{ }^{\circ} \mathrm{C}$ in a heat bath; this cycle was repeated until the lipids dissolved. For 20Z pMMO, a pMMO trimer:MSP2N2:POPC molar ratio of 1:13:2340 was used for $5-10 \mathrm{~mL}$ reconstitutions in $20 \mathrm{mM}$ PIPES, pH $7.3,250 \mathrm{mM} \mathrm{NaCl}, 0.02 \% \mathrm{DDM}$ buffer. The final concentration of the components in the reconstitution mixture was $3.33 \mu \mathrm{M}$ pMMO trimer, $43.3 \mu \mathrm{M}$ MSP2N2, $7.8 \mathrm{mM}$ POPC, and $20 \mathrm{mM}$ sodium cholate. For 20Z-pMMO using MSP1E3D1, the reconstitution molar ratio of pMMO:MSP1E3D1:POPC was 1:13:390 with final concentrations of $3.33 \mu \mathrm{M}$ pMMO trimer, $43.3 \mu \mathrm{M}$ MSP1E3D1, $1.3 \mathrm{mM}$ POPC, and $20 \mathrm{mM}$ sodium cholate. For Rockwell-pMMO using MSP1E3D1, the molar ratio of pMMO:MSP1E3D1: POPC was also 1:13:390. The reconstitution mixtures were rotated on a tube rotator at $4{ }^{\circ} \mathrm{C}$ for $1 \mathrm{~h}$, followed by an overnight dialysis at $4{ }^{\circ} \mathrm{C}$ using a $10 \mathrm{kDa}$ MWCO Slide-A-Lyzer dialysis cassette (ThermoFisher). For 20Z-pMMO, the reconstituted mixture was buffer exchanged into $20 \mathrm{mM}$ PIPES, $\mathrm{pH} 7.3,50 \mathrm{mM}$ $\mathrm{NaCl}$, and loaded onto a $5 \mathrm{~mL}$ or $10 \mathrm{~mL}$ HiTrap Q FF anion exchange chromatography column (GE Healthcare). A $50-800 \mathrm{mM} \mathrm{NaCl}$ gradient was used to separate empty nanodiscs and pMMO-embedded nanodiscs. Empty nanodiscs eluted at $300 \mathrm{mM} \mathrm{NaCl}$, and 20Z-pMMO nanodiscs eluted at $600 \mathrm{mM} \mathrm{NaCl}$. pMMO-nanodisc fractions were collected and concentrated using a $100 \mathrm{kDa}$ MWCO Amicon centrifugal concentrator (Millipore). The 20Z-pMMO (post anion exchange) samples were loaded onto a Superose 6 Increase 10/300 GL column (GE Healthcare). Fractions corresponding to the pMMO-nanodisc complex were collected and concentrated using a using a $100 \mathrm{kDa}$ MWCO Amicon centrifugal concentrator (Millipore). Protein concentrations of 20Z-pMMO nanodisc samples were measured using the DC Lowry assay with BSA as a standard. Copper content of 20Z-pMMO samples was determined using inductively coupled plasma optical emission spectrometry (ICP-OES), and copper content of Rockwell-pMMO samples was determined by ICP-MS, both at Northwestern University's Quantitative Bio-element Imaging Center (QBIC). Copper concentrations were quantified using $0-500 \mathrm{ppb}$ copper standards (Inorganic Ventures).

pMMO reconstitution into nanodiscs using Bio-Beads. For copper supplementation experiments, Rockwell-pMMO was reconstituted into MSP1E3D1 nanodiscs using Bio-Beads (Bio-Rad). pMMO, MSP1E3D1, and POPC were mixed together for $2 \mathrm{~h}$ at $4{ }^{\circ} \mathrm{C}$ at a molar ratio of 1:4:240 pMMO:MSP1E3D1:POPC. For copper supplementation experiments, one molar equivalent of $\mathrm{CuSO}_{4} \cdot 5 \mathrm{H}_{2} \mathrm{O}$ per solubilized Rockwell-pMMO protomer was added into the mixture. Self-assembly of the nanodisc was initiated by adding $0.5 \mathrm{~g} / \mathrm{mL}$ wet Bio-Beads to the mixture followed by rotating on a tube rotator for $2 \mathrm{~h}$ at $4{ }^{\circ} \mathrm{C}$. Wet Bio-Beads were prepared by mixing dry Bio-Beads with $25 \mathrm{mM}$ PIPES, pH 7.3, $250 \mathrm{mM} \mathrm{NaCl}$. Wet Bio-Beads were weighed by decanting onto a weigh boat followed by removal of the excess liquid with a Pasteur pipet. After reconstitution, Bio-Beads were removed from the nanodisc mixture by passing the mixture through a $0.22 \mu \mathrm{m}$ syringe filter. The nanodiscs were then concentrated and purified by size-exclusion chromatography using a Superose 6 Increase 10/300 GL column (GE Healthcare). Fractions were collected and concentrated using a $100 \mathrm{kDa}$ MWCO Amicon centrifugal concentrator (Millipore).

Empty nanodiscs could not be separated from the Rockwell-pMMO nanodisc samples, so the DC-Lowry Assay could not be used for accurate protein concentration measurements. Instead, Rockwell-pMMO nanodisc complexes were quantified using SDS-PAGE and ImageJ software ${ }^{53}$. Solubilized Rockwell-pMMO of known concentration (measured using the DC Lowry assay, Bio-Rad) was loaded onto a $15 \%$ SDS-PAGE gel at concentrations of 4,2 , and $1 \mathrm{mg} / \mathrm{mL}$. ImageJ was then used to generate a standard curve, correlating the intensity of the PmoB subunit band with the known concentrations. The PmoB subunit was used for the standard curve since it was well separated from the PmoA, PmoC, and MSP bands on the gel. The concentration of the Rockwell-pMMO nanodisc sample was measured by comparing the intensity of its PmoB band against the standard curve generated above. Copper content was determined using inductively coupled plasma mass spectrometry (ICP-MS) at Northwestern University's Quantitative Bioelement Imaging Center (QBIC). Copper concentrations were quantified using 0-500 ppb copper standards (Inorganic Ventures).

Cryo-EM sample preparation and data acquisition. Freshly purified 20Z-pMMO sample in MSP2N2 nanodisc $(3 \mu \mathrm{L}$ at $\sim 0.5 \mathrm{mg} / \mathrm{mL})$ was deposited onto glowdischarged 400 mesh 1.2/1.3 C-Flat grids (Protochips). The Vitrobot Mark IV (FEI) sample chamber was kept at $100 \%$ relative humidity and the grid was blotted for $5-8 \mathrm{~s}$ before plunge freezing in a liquid ethane bath cooled by liquid nitrogen. The grids were imaged using a JEOL 3200FS microscope operating at $300 \mathrm{kV}$. Data were acquired on a K2 summit camera (Gatan) using Leginon ${ }^{54}$ with a defocus range between $1.5-3.5 \mu \mathrm{m}$ using counting mode with a pixel size of $1.1 \AA$. Movies were recorded for $6 \mathrm{~s}$ exposure with a dose rate of approximately $8 \mathrm{e}-/ \mathrm{pix} / \mathrm{s}$ (equivalent to $6.6 \mathrm{e}-/ \AA^{2} / \mathrm{s}$ on the plane of the sample).

Recorded movies were subjected to gain correction and then beam-induced motion correction with MotionCor ${ }^{55}$. Following contrast transfer function (CTF) estimation with CTFFIND $4^{56}$, micrographs with the best quality were then selected for further processing. Particles were then picked, extracted, and classified for $2 \mathrm{D}$ classification by applying C3 symmetry using the Scipion ${ }^{57}$ software environment using XMIPP programs ${ }^{58,59}$. Three best $2 \mathrm{D}$ classes representing different orientations of $20 \mathrm{Z}$-pMMO in nanodiscs are shown in Fig. 4. 
${ }^{13} \mathrm{C}$ methane oxidation activity assay. Methane oxidation activity levels of pMMO-nanodisc complexes were performed ${ }^{4}$. Rockwell-pMMO in nanodiscs $(\sim 2-4 \mathrm{mg} / \mathrm{mL})$ was resuspended in $25 \mathrm{mM}$ PIPES, $\mathrm{pH} 7.2,250 \mathrm{mM} \mathrm{NaCl}$ in $100 \mu \mathrm{L}$ reactions containing reductant (excess duroquinol) in $2 \mathrm{~mL}$ screw top vials sealed with septa (Agilent). A $1 \mathrm{~mL}$ volume of headspace gas was withdrawn from the reaction vial and replaced with $1.5 \mathrm{~mL}$ of ${ }^{13} \mathrm{C}$ methane gas (Sigma-Aldrich). All reactions were performed at $30^{\circ} \mathrm{C}$ and $200 \mathrm{rpm}$ for $5 \mathrm{~min}$. The reactions were placed on ice for $5 \mathrm{~min}$ followed by quenching with $500 \mu \mathrm{L}$ of chloroform containing $1 \mathrm{mM}$ dichloromethane. The reaction was vortexed at $2000 \mathrm{rpm}$ for $10 \mathrm{~min}$ and centrifuged at $2,000 \times g$ for $30 \mathrm{~min}$. $2.5 \mu \mathrm{L}$ of the chloroform mixture was injected into a PoraBOND Q column $(25 \mathrm{~m}$ x $250 \mu \mathrm{m} \times 3 \mu \mathrm{m})$ on an Agilent 7890B/5977A MSD GC/MS instrument with a split ratio of 10:1. The column was under a constant flow of $1.2 \mathrm{~mL} / \mathrm{min}$ of helium gas. The GC protocol was as follows: oven temperature was maintained at $80^{\circ} \mathrm{C}$ for $3.5 \mathrm{~min}$, ramped $50^{\circ} \mathrm{C} / \mathrm{min}$ to $150{ }^{\circ} \mathrm{C}$ and held for $1.5 \mathrm{~min}$, and then ramped $15^{\circ} \mathrm{C} / \mathrm{min}$ to $300^{\circ} \mathrm{C}$ and held for $1 \mathrm{~min}$. The MS instrument protocol was as follows: $230^{\circ} \mathrm{C}$ ion source temperature, $150^{\circ} \mathrm{C}$ quad temperature, $70 \mathrm{eV}$, and a detector voltage of $2999 \mathrm{~V}$. Ion masses 31 , 33 , and 49 were monitored for detection of ${ }^{12} \mathrm{C}$ methanol, ${ }^{13} \mathrm{C}$ methanol, and dichloromethane with dwell times of 10,100 , and $10 \mathrm{~ms}$, respectively. ${ }^{13} \mathrm{C}$ methanol concentrations were quantified using a standard calibration curve and the dichloromethane internal standard.

\section{Native mass spectrometry analysis. pMMO-nanodiscs samples for nTDMS} analysis were dialyzed overnight using $10 \mathrm{kDa}$ MWCO Slide-A-Lyzer MINI dialysis devices (Thermo Scientific) into $200 \mathrm{mM}$ ammonium acetate, pH 7.2 (adjusted using ammonium hydroxide), and concentrated to approximately $30 \mu \mathrm{M}$ pMMOnanodisc complex. For detergent-solubilized pMMO samples, pMMO solubilized in DDM detergent were buffer exchanged into $200 \mathrm{mM}$ ammonium acetate, $\mathrm{pH}$ 7.2, $0.155 \%(\mathrm{w} / \mathrm{v})$ Triton X-100. Samples were analyzed using a Q Exactive HF mass spectrometer with Extended Mass Range and data were collected using XCalibur QualBrowser 4.0.27.10 (Thermo Fisher Scientific, Waltham, MA). The nTDMS platform employs direct infusion of sample into a native electrospray ionization (nESI) source held at $+2 \mathrm{kV}$, C-trap entrance lens voltage setting between $1.8-4 \mathrm{~V}, \mathrm{HCD}$ gas pressure setting between $2-4 \mathrm{~V}$, and CID voltage set at $50-100 \mathrm{~V}$ for desalting and $150-195 \mathrm{~V}$ for protein ejection from detergent micelles or nanodisc complexes. The nTDMS platform is coupled to a three-tiered tandem MS process. The first step in the process ${ }^{19}$ is the analysis of the intact protein complex $\left(\mathrm{MS}^{1}\right)$, which provides the total mass (reported as a deconvoluted neutral average mass value). In stage two, the complex is collisionally activated with nitrogen gas to eject monomers $\left(\mathrm{MS}^{2}\right)$, thereby liberating the subunits that comprise each intact complex. In stage three, further vibrational activation of the ejected subunits via collisions with nitrogen gas yields backbone fragmentation products from each monomer $\left(\mathrm{MS}^{3}\right)$ that are recorded at isotopic resolution (120,000 resolving power at $\mathrm{m} / \mathrm{z} 400)$. These fragments are used to characterize the primary sequence of the monomers and localize posttranslational modifications. Intact mass values for pMMO complexes and ejected subunits, the MS ${ }^{1}$ and $\mathrm{MS}^{2}$ meassurements, were determined by deconvolution to convert data from the $\mathrm{m} / \mathrm{z}$ to the mass domain using MagTran $1.03^{60}$ (mass range: 15,000-300,000 Da; max number of species: $10-15$; S/N threshold: 1; mass accuracy: $0.05 \mathrm{Da}$; charge determined by: charge envelope only). Intact mass measurements are reported as neutral average masses; errors represent $1 \sigma$ deviation from the mean of the masses calculated for of all sampled charge states. Fragmentation data were processed using mMass 5.5.0 (www.mmass.org), ProSight Lite 1.4 ${ }^{38}$ (precursor mass type: average; fragmentation method: HCD; fragmentation tolerance: $25 \mathrm{ppm}$ ), and TDValidator $1.0^{61}$ (max ppm tolerance: $25 \mathrm{ppm}$; cluster tolerance: 0.35 ; charge range: $1-10$; minimum score: $0.5 ; \mathrm{S} / \mathrm{N}$ cutoff: 3 ; Mercury7 limit: 0.0001 ; minimum size: 2) to assign recorded fragment ions to the primary sequence of the subunits. Specifically, ProSight Lite and TDValidator were used to analyze fragmentation spectra in medium throughput to assign and validate $b$ and $y$ fragment ions to the pMMO subunit sequences, and for generating a p-score. mMass was used to interrogate individual fragment ions within a spectrum, for example to examine metal binding and to validate that fragment ions correspond to their theoretical mass. The PmoA, PmoB, and PmoC subunits of 20Z-pMMO were identified by mapping backbone fragment ions to the amino acid sequence of pMMO subunits using ProSight Lite, with the p-scores ${ }^{38}$ of $3.2 \times 10^{-39}$ (PmoA), $6 \times 10^{-15}(\mathrm{PmoB})$, and $3.7 \times 10^{-14}(\mathrm{PmoC})$. The PmoB and PmoC subunits of Rockwell-pMMO were identified using ProSight Lite with p-scores of $3.5 \times 10^{-8}$ and $1.6 \times 10^{-34}$, respectively ${ }^{62}$. Unexplained mass shifts $(\Delta \mathrm{m})$ observed at the $\mathrm{MS}^{1}, \mathrm{MS}^{2}$, and $\mathrm{MS}^{3}$ levels for the intact complex and subunits, respectively, were manually interrogated using the UNIMOD database (http://www.unimod.org/modifications_list.php) as a reference for candidate modifications.

Bottom-up proteomics methods. pMMO subunits from solubilized 20Z- and 5GpMMO samples were separated using a reverse-phase HPLC 214TP54 analytical C4 column (Grace Vydac) on an Agilent 1100 HPLC $^{48}$. Briefly, $100 \mu \mathrm{L}$ of $30 \mu \mathrm{M}$ pMMO trimer was injected onto the column and eluted using a gradient from $100 \%$ solvent A ( $63.75 \%$ formic acid, $10 \%$ acetonitrile, $5 \% i$ - PrOH) to $100 \%$ solvent B (70\% formic acid, 30\% $i-\mathrm{PrOH})$. Eluted fractions were diluted 1:8 with water and digested with $2 \mu \mathrm{g}$ pepsin (Promega) overnight at $37^{\circ} \mathrm{C}$. Pepsin was inactivated by heating samples at $95^{\circ} \mathrm{C}$ for $10 \mathrm{~min}$. The peptides were desalted on a C18 spin column (Pierce), and eluted with $80 \%$ acetonitrile in $0.1 \%$ formic acid. Samples were then lyophilized, resuspended with $5 \%$ acetonitrile in $0.1 \%$ formic acid, and injected onto a trap column $(150 \mu \mathrm{m}$ i.d. $\times 3 \mathrm{~cm})$ coupled with a nanobore analytical column $(75 \mu \mathrm{m}$ i.d. $\times 15 \mathrm{~cm}$, both ReproSil C18aq, $3 \mu \mathrm{m})$. Samples were separated using a linear gradient of solvent A (95\% water, $5 \%$ acetonitrile, $0.1 \%$ formic acid) and solvent B (5\% water, $95 \%$ acetonitrile, $0.1 \%$ formic acid). MS data were collected using a Velos Orbitrap Elite (Thermo) mass spectrometer operating in data-dependent top 10 mode. MS ${ }^{1}$ data were collected at a resolution of 60,000 at $m / z 400$ and an AGC target of 1,000,000. MS 2 data were collected from the top 10 peaks in each precursor scan isolated with a $1.5 \mathrm{~m} / z$ isolation width fragmenting with CID with a normalized collision energy of 35 at an activation q of 0.25 and a duration of $10 \mathrm{~ms}$ at an AGC target of 10,000. Fragment ion spectra were recorded in the ion trap. The collected data were searched using Mascot 2.5 (Matrix Science) against custom proteomic databases for $\mathrm{Mm}$. buryatense $5 \mathrm{G}$ and $\mathrm{Mm}$. alcaliphilum $20 \mathrm{Z}$ constructed from their published genomes ${ }^{63,64}$. Peptide $\mathrm{MS}^{1}$ tolerance was 15 $\mathrm{ppm}$, while $\mathrm{MS}^{2}$ tolerance was $0.6 \mathrm{Da}$, and no cleavage enzyme was selected, so all subsequences for each protein in the database were queried. Variable modifications of deamidation of asparagine/glutamine and oxidation of methionine were allowed Peptide fragmentation data were reported at $1 \%$ false discovery rate in Scaffold 4.5 (Proteome Software). Peptides containing post-translational modifications were validated by manual inspection of the tandem MS data. Bottom-up data proteomics have been deposited in the MASSive database with accession number MSV000083717 [https://doi.org/10.25345/C5ND0J].

Reporting summary. Further information on research design is available in the Nature Research Reporting Summary linked to this article.

\section{Data availability}

Bottom-up proteomics data have been deposited in the MASSive database with accession number MSV000083717 [https://doi.org/10.25345/C5ND0J]. Supplementary Data 1 contains expected and experimentally determined masses for nTDMS experiments. The source data underlying Fig. 7a, b and Supplementary Figs. 4 and 8 are provided as a Source Data file. A reporting summary for this Article is available as a Supplementary Information file. All other data supporting the findings of this study are available from the corresponding authors on reasonable request.

Received: 6 February 2019 Accepted: 15 May 2019

Published online: 17 June 2019

\section{References}

1. Sirajuddin, S. \& Rosenzweig, A. C. Enzymatic oxidation of methane. Biochemistry 54, 2283-2294 (2015).

2. Hanson, R. S. \& Hanson, T. E. Methanotrophic bacteria. Microbiol. Rev. 60, 439-471 (1996).

3. Sirajuddin, S. et al. Effects of zinc on particulate methane monooxygenase activity and structure. J. Biol. Chem. 289, 21782-21794 (2014).

4. Ro, S. Y. et al. From micelles to bicelles: effect of the membrane on particulate methane monooxygenase activity. J. Biol. Chem. 293, 10457-10465 (2018).

5. Smith, S. M. et al. Crystal structure and characterization of particulate methane monooxygenase from Methylocystis species strain M. Biochemistry 50, 10231-10240 (2011).

6. Lieberman, R. L. \& Rosenzweig, A. C. Crystal structure of a membrane-bound metalloenzyme that catalyses the biological oxidation of methane. Nature $\mathbf{4 3 4}$, 177-182 (2005).

7. Hakemian, A. S. et al. The metal centers of particulate methane monooxygenase from Methylosinus trichosporium OB3b. Biochemistry 47, 6793-6801 (2008)

8. Balasubramanian, R. et al. Oxidation of methane by a biological dicopper centre. Nature 465, 115-119 (2010).

9. Wang, V. C. et al. Alkane oxidation: methane monooxygenases, related enzymes, and their biomimetics. Chem. Rev. 117, 8574-8621 (2017).

10. Lawton, T. J. \& Rosenzweig, A. C. Methane-oxidizing enzymes: an upstream problem in biological gas-to-liquids conversion. J. Am. Chem. Soc. 138, 9327-9340 (2016)

11. Kalyuzhnaya, M. G., Puri, A. W. \& Lidstrom, M. E. Metabolic engineering in methanotrophic bacteria. Metab. Eng. 29, 142-152 (2015).

12. Lieberman, R. L. et al. Characterization of the particulate methane monooxygenase metal centers in multiple redox states by X-ray absorption spectroscopy. Inorg. Chem. 45, 8372-8381 (2006).

13. Cao, L. L., Caldararu, O., Rosenzweig, A. C. \& Ryde, U. Quantum refinement does not support dinuclear copper sites in crystal structures of particulate methane monooxygenase. Angew. Chem. Int. Ed. 57, 162-166 (2018).

14. Ross, M. O. et al. Particulate methane monooxygenase contains only mononuclear copper centers. Science 364, 566-570 (2019). 
15. Leney, A. C. \& Heck, A. J. Native mass spectrometry: what is in the name? J. Am. Soc. Mass Spectrom. 28, 5-13 (2017).

16. Loo, J. A. Studying noncovalent protein complexes by electrospray ionization mass spectrometry. Mass Spectrom. Rev. 16, 1-23 (1997).

17. Loo, J. A. Electrospray ionization mass spectrometry: a technology for studying noncovalent macromolecular complexes. Int. J. Mass Spectrom. 200, 175-186 (2000).

18. Benesch, J. L., Aquilina, J. A., Ruotolo, B. T., Sobott, F. \& Robinson, C. V. Tandem mass spectrometry reveals the quaternary organization of macromolecular assemblies. Chem. Biol. 13, 597-605 (2006).

19. Belov, M. E. et al. From protein complexes to subunit backbone fragments: a multi-stage approach to native mass spectrometry. Anal. Chem. 85, 11163-11173 (2013).

20. Skinner, O. S. et al. An informatic framework for decoding protein complexes by top-down mass spectrometry. Nat. Methods 13, 237-240 (2016).

21. Smith, L. M. et al. Proteoform: a single term describing protein complexity. Nat. Methods 10, 186-187 (2013).

22. Haverland, N. A. et al. Defining gas-phase fragmentation propensities of intact proteins during native top-down mass spectrometry. J. Am. Soc. Mass Spectrom. 28, 1203-1215 (2017).

23. Skinner, O. S., Schachner, L. F. \& Kelleher, N. L. The search engine for multiproteoform complexes: an online tool for the identification and stoichiometry determination of protein complexes. Curr. Protoc. Bioinformatics 56, 13.30.11-13.30.11 (2016).

24. Li, H., Nguyen, H. H., Ogorzalek Loo, R. R., Campuzano, I. D. G. \& Loo, J. A. An integrated native mass spectrometry and top-down proteomics method that connects sequence to structure and function of macromolecular complexes. Nat. Chem. 10, 139-148 (2018)

25. Skinner, O. S. et al. Top-down characterization of endogenous protein complexes with native proteomics. Nat. Chem. Biol. 14, 36-41 (2017).

26. Kenney, G. E. et al. The biosynthesis of methanobactin. Science 359, 1411-1416 (2018).

27. Wongkongkathep, P. et al. Native top-down mass spectrometry and ion mobility MS for characterizing the cobalt and manganese metal binding of $\alpha$ synuclein protein. J. Am. Soc. Mass Spectrom. 29, 1870-1880 (2018).

28. Gault, J. et al. High-resolution mass spectrometry of small molecules bound to membrane proteins. Nat. Methods 13, 333 (2016).

29. Hopper, J. T. S. et al. Detergent-free mass spectrometry of membrane protein complexes. Nat. Methods 10, 1206-1208 (2013).

30. Laganowsky, A., Reading, E., Hopper, J. T. S. \& Robinson, C. V. Mass spectrometry of intact membrane protein complexes. Nat. Protoc. 8, 639-651 (2013).

31. Marty, M. T., Hoi, K. K., Gault, J. \& Robinson, C. V. Probing the lipid annular belt by gas-phase dissociation of membrane proteins in nanodiscs. Angew. Chem. Int. Ed. Engl. 55, 550-554 (2016)

32. Grinkova, Y. V., Denisov, I. G. \& Sligar, S. G. Engineering extended membrane scaffold proteins for self-assembly of soluble nanoscale lipid bilayers. Protein Eng. Des. Sel. 23, 843-848 (2010).

33. Chistoserdova, L. \& Kalyuzhnaya, M. G. Current trends in methylotrophy. Trends Microbiol. 26, 703-714 (2018).

34. Akberdin, I. R. et al. Methane utilization in Methylomicrobium alcaliphilum 20Z(R): a systems approach. Sci. Rep. 8, 2512 (2018).

35. Nguyen, A. D. et al. Systematic metabolic engineering of Methylomicrobium alcaliphilum $20 \mathrm{Z}$ for 2,3-butanediol production from methane. Metab. Eng. 47, 323-333 (2018).

36. Semrau, J. D. et al. Particulate methane monooxygenase genes in methanotrophs. J. Bacteriol. 177, 3071-3079 (1995).

37. Denisov, I. G. \& Sligar, S. G. Nanodiscs in membrane biochemistry and biophysics. Chem. Rev. 117, 4669-4713 (2017).

38. Fellers, R. T. et al. ProSight Lite: graphical software to analyze top-down mass spectrometry data. Proteomics 15, 1235-1238 (2015).

39. Yu, S. S. F. et al. The C-terminal aqueous-exposed domain of the $45 \mathrm{kDa}$ subunit of the particulate methane monooxygenase in Methylococcus capsulatus (Bath) is a $\mathrm{Cu}(\mathrm{I})$ sponge. Biochemistry 46, 13762-13774 (2007).

40. Kahnt, J. et al. Post-translational modifications in the active site region of methyl-coenzyme $\mathrm{M}$ reductase from methanogenic and methanotrophic archaea. FEBS J. 274, 4913-4921 (2007).

41. Petrović, D. M. et al. Methylation of the $\mathrm{N}$-terminal histidine protects a lytic polysaccharide monooxygenase from auto-oxidative inactivation. Protein Sci. 27, 1636-1650 (2018).

42. Nyyssölä, A., Kerovuo, J., Kaukinen, P., von Weymarn, N. \& Reinikainen, T. Extreme halophiles synthesize betaine from glycine by methylation. J. Biol. Chem. 275, 22196-22201 (2000)

43. Liew, E. F., Tong, D., Coleman, N. V. \& Holmes, A. J. Mutagenesis of the hydrocarbon monooxygenase indicates a metal centre in subunit- $\mathrm{C}$, and not subunit- $\mathrm{B}$, is essential for copper-containing membrane monooxygenase activity. Microbiology 160, 1267-1277 (2014).
44. Cowley, R. E., Tian, L. \& Solomon, E. I. Mechanism of O2 activation and substrate hydroxylation in noncoupled binuclear copper monooxygenases. Proc. Natl Acad. Sci. USA 113, 12035-12040 (2016).

45. Op den Camp, H. J. et al. Environmental, genomic and taxonomic perspectives on methanotrophic Verrucomicrobia. Environ. Microbiol. Rep. 1, 293-306 (2009).

46. Hakemian, A. S. \& Rosenzweig, A. C. The biochemistry of methane oxidation. Annu. Rev. Biochem. 76, 223-241 (2007).

47. Miller, K. W., Hammond, L. \& Porter, E. G. Solubility of hydrocarbon gases in lipid bilayers. Chem. Phys. Lipids 20, 229-241 (1977).

48. Fisher, O. S. et al. Characterization of a long overlooked copper protein from methane- and ammonia-oxidizing bacteria. Nat. Commun. 9, 4276 (2018).

49. Lieven, C. et al. A genome-scale metabolic model for Methylococcus capsulatus (Bath) suggests reduced efficiency electron transfer to the particulate methane monooxygenase. Front. Microbiol. 9, 2947 (2018).

50. Makula, R. A. Phospholipid composition of methane-oxidizing bacteria. J. Bacteriol. 134, 771-777 (1978).

51. Chorev, D. S. et al. Protein assemblies ejected directly from native membranes yield complexes for mass spectrometry. Science 362, 829-834 (2018).

52. Deng, Y. W., Ro, S. Y. \& Rosenzweig, A. C. Structure and function of the lanthanide-dependent methanol dehydrogenase XoxF from the methanotroph Methylomicrobium buryatense 5GB1C. J. Biol. Inorg. Chem. 23, 1037-1047 (2018).

53. Schneider, C. A., Rasband, W. S. \& Eliceiri, K. W. NIH image to ImageJ: 25 years of image analysis. Nat. Methods 9, 671-675 (2012).

54. Suloway, C. et al. Automated molecular microscopy: the new Leginon system. J. Struct. Biol. 151, 41-60 (2005).

55. Zheng, S. Q. et al. MotionCor2: anisotropic correction of beam-induced motion for improved cryo-electron microscopy. Nat. Methods 14, 331-332 (2017).

56. Rohou, A. \& Grigorieff, N. CTFFIND4: fast and accurate defocus estimation from electron micrographs. J. Struct. Biol. 192, 216-221 (2015).

57. de la Rosa-Trevín, J. M. et al. Scipion: a software framework toward integration, reproducibility and validation in $3 \mathrm{D}$ electron microscopy. $J$. Struct. Biol. 195, 93-99 (2016).

58. Abrishami, V. et al. A pattern matching approach to the automatic selection of particles from low-contrast electron micrographs. Bioinformatics 29, 2460-2468 (2013).

59. Sorzano, C. O. et al. A clustering approach to multireference alignment of single-particle projections in electron microscopy. J. Struct. Biol. 171, 197-206 (2010).

60. Zhang, Z. \& Marshall, A. G. A universal algorithm for fast and automated charge state deconvolution of electrospray mass-to-charge ratio spectra. J. Am. Soc. Mass Spectrom. 9, 225-233 (1998).

61. Fornelli, L. et al. Accurate sequence analysis of a monoclonal antibody by topdown and middle-down orbitrap mass spectrometry applying multiple Ion activation techniques. Anal. Chem. 90, 8421-8429 (2018).

62. Stein, L. Y. et al. Genome sequence of the methanotrophic alphaproteobacterium Methylocystis sp. strain Rockwell (ATCC 49242). J. Bacteriol. 193, 2668-2669 (2011).

63. Vuilleumier, S. et al. Genome sequence of the haloalkaliphilic methanotrophic bacterium Methylomicrobium alcaliphilum 20Z. J. Bacteriol. 194, 551-552 (2012).

64. Khmelenina, V. N. et al. Draft genome sequence of Methylomicrobium buryatense strain $5 \mathrm{G}$, a haloalkaline-tolerant methanotrophic bacterium. Genome Annouc. 1, e00053-00013 (2013).

65. Cassou, C. A. \& Williams, E. R. Desalting protein ions in native mass spectrometry using supercharging reagents. Analyst 139, 4810-4819 (2014).

\section{Acknowledgements}

This work was funded by National Institutes of Health grants GM118035 (A.C.R.) and 1S10OD025194-01 (N.L.K). Research in this publication is also supported by a fellowship associated with the Chemistry of Life Processes Predoctoral Training Grant T32GM105538 at Northwestern University. L.F.S. is a Gilliam Fellow of the Howard Hughes Medical Institute. The Quantitative Bio-element Imaging Center at Northwestern is supported by NASA Ames Research Center NNA06CB93G. The Proteomics Center for Excellence and at Northwestern is supported by NIH grant NCI CCSG P30 CA060553. The support from the R.H. Lurie Comprehensive Cancer Center of Northwestern University to the Northwestern University Structural Biology Facility is acknowledged. The Gatan K2 direct electron detector was purchased with funds provided by the Chicago Biomedical Consortium with support from the Searle Funds at The Chicago Community Trust. Collection of electron microscopy data was supported by Chicago Biomedical Consortium Postdoctoral Research Award PDR-097 (R. P.) with support from the Searle Funds at The Chicago Community Trust. 


\section{Author contributions}

S.Y.R. was responsible for the preparation and biochemical analysis of pMMO and the nanodisc complexes, while L.F.S. collected and analyzed all the nTDMS data. C.W.K. was responsible for the metal content analysis and methane oxidation activity measurements of Rockwell-pMMO. R.P. contributed to the EM analysis of pMMO-nanodisc complexes and collected cryo-EM data of 20Z-pMMO. J.P.R. processed the cryo-EM data of 20ZpMMO to produce $2 \mathrm{D}$ class averages. G.E.K. and P.M.T. provided the bottom-up MS data on 20Z-, 5G-, and Rockwell-pMMO. B.W.L. helped with the initial optimization of 20Z-pMMO reconstitution into nanodiscs. S.M.P. provided key insights and revisions in the editing of the manuscript. A.C.R. and N.L.K. contributed heavily to experimental design and writing of the manuscript.

\section{Additional information}

Supplementary Information accompanies this paper at https://doi.org/10.1038/s41467019-10590-6.

Competing interests: N.L.K. is a consultant for Thermo Fisher Scientific. The remaining authors declare no competing interests.

Reprints and permission information is available online at http://npg.nature.com/ reprintsandpermissions/
Peer review information: Nature Communications thanks the anonymous reviewers for their contribution to the peer review of this work.

Publisher's note: Springer Nature remains neutral with regard to jurisdictional claims in published maps and institutional affiliations.

(c) (i) Open Access This article is licensed under a Creative Commons Attribution 4.0 International License, which permits use, sharing, adaptation, distribution and reproduction in any medium or format, as long as you give appropriate credit to the original author(s) and the source, provide a link to the Creative Commons license, and indicate if changes were made. The images or other third party material in this article are included in the article's Creative Commons license, unless indicated otherwise in a credit line to the material. If material is not included in the article's Creative Commons license and your intended use is not permitted by statutory regulation or exceeds the permitted use, you will need to obtain permission directly from the copyright holder. To view a copy of this license, visit http://creativecommons.org/ licenses/by/4.0/.

(C) The Author(s) 2019 